\title{
An extremely luminous and variable ultraluminous $x$-ray source in the outskirts of circinus observed with NuSTAR
}

\author{
Walton, D. J.; Fuerst, F.; Harrison, F.; Stern, D.; Bachetti, M.; Barret, D.; Bauer, F.; Boggs, S. E.; \\ Christensen, Finn Erland; Craig, W. W.
}

Total number of authors:

19

Published in:

Astrophysical Journal

Link to article, DOI:

10.1088/0004-637X/779/2/148

Publication date:

2013

Document Version

Publisher's PDF, also known as Version of record

Link back to DTU Orbit

Citation (APA):

Walton, D. J., Fuerst, F., Harrison, F., Stern, D., Bachetti, M., Barret, D., Bauer, F., Boggs, S. E., Christensen, F. E., Craig, W. W., Fabian, A. C., Grefenstette, B. W., Hailey, C. J., Madsen, K. K., Miller, J. M., Ptak, A., Rana, V., Webb, N. A., \& Zhang, W. W. (2013). An extremely luminous and variable ultraluminous x-ray source in the outskirts of circinus observed with NuSTAR. Astrophysical Journal, 779(2), [145]. https://doi.org/10.1088/0004$637 \mathrm{X} / 779 / 2 / 148$

\section{General rights}

Copyright and moral rights for the publications made accessible in the public portal are retained by the authors and/or other copyright owners and it is a condition of accessing publications that users recognise and abide by the legal requirements associated with these rights.

- Users may download and print one copy of any publication from the public portal for the purpose of private study or research.

- You may not further distribute the material or use it for any profit-making activity or commercial gain

- You may freely distribute the URL identifying the publication in the public portal 


\title{
AN EXTREMELY LUMINOUS AND VARIABLE ULTRALUMINOUS X-RAY SOURCE IN THE OUTSKIRTS OF CIRCINUS OBSERVED WITH NUSTAR
}

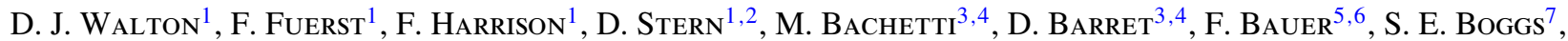 \\ F. E. Christensen ${ }^{8}$, W. W. Craig ${ }^{7}, 9$, A. C. Fabian $^{10}$, B. W. Grefenstette ${ }^{1}$, C. J. Hailey ${ }^{11}$, K. K. Madsen ${ }^{1}$, \\ J. M. MilleR ${ }^{12}$, A. PtaK ${ }^{13}$, V. RANA ${ }^{1}$, N. A. WebB ${ }^{3,4}$, AND W. W. ZhanG ${ }^{13}$ \\ ${ }^{1}$ Space Radiation Laboratory, California Institute of Technology, Pasadena, CA 91125, USA \\ 2 Jet Propulsion Laboratory, California Institute of Technology, Pasadena, CA 91109, USA \\ ${ }^{3}$ Universite de Toulouse, UPS-OMP, IRAP, Toulouse, France \\ ${ }^{4}$ CNRS, IRAP, 9 Av. colonel Roche, BP 44346, F-31028 Toulouse cedex 4, France \\ ${ }^{5}$ Instituto de Astrofísica, Facultad de Física, Pontificia Universidad Católica de Chile, 306, Santiago 22, Chile \\ ${ }^{6}$ Space Science Institute, 4750 Walnut Street, Suite 205, Boulder, CO 80301, USA \\ ${ }^{7}$ Space Sciences Laboratory, University of California, Berkeley, CA 94720, USA \\ ${ }^{8}$ DTU Space, National Space Institute, Technical University of Denmark, Elektrovej 327, DK-2800 Lyngby, Denmark \\ ${ }^{9}$ Lawrence Livermore National Laboratory, Livermore, CA 94550, USA \\ ${ }^{10}$ Institute of Astronomy, University of Cambridge, Madingley Road, Cambridge CB3 0HA, UK \\ ${ }^{11}$ Columbia Astrophysics Laboratory, Columbia University, New York, NY 10027, USA \\ 12 Department of Astronomy, University of Michigan, 500 Church Street, Ann Arbor, MI 48109-1042, USA \\ ${ }^{13}$ NASA Goddard Space Flight Center, Greenbelt, MD 20771, USA \\ Received 2013 August 18; accepted 2013 October 8; published 2013 December 3
}

\begin{abstract}
Following a serendipitous detection with the Nuclear Spectroscopic Telescope Array (NuSTAR), we present a multiepoch spectral and temporal analysis of an extreme ultraluminous X-ray source (ULX) located in the outskirts of the Circinus galaxy, hereafter Circinus ULX5, including coordinated XMM-Newton+NuSTAR follow-up observations. The NUSTAR data presented here represent one of the first instances of a ULX reliably detected at hard $(E>10 \mathrm{keV})$ X-rays. Circinus ULX5 is variable on long time scales by at least a factor of $\sim 5$ in flux, and was caught in a historically bright state during our 2013 observations $\left(0.3-30.0 \mathrm{keV}\right.$ luminosity of $\left.1.6 \times 10^{40} \mathrm{erg} \mathrm{s}^{-1}\right)$. During this epoch, the source displayed a curved 3-10 keV spectrum, broadly similar to other bright ULXs. Although pure thermal models result in a high energy excess in the NUSTAR data, this excess is too weak to be modeled with the disk reflection interpretation previously proposed to explain the 3-10 keV curvature in other ULXs. In addition to flux variability, clear spectral variability is also observed. While in many cases the interpretation of spectral components in ULXs is uncertain, the spectral and temporal properties of all the high quality data sets currently available strongly support a simple disk-corona model reminiscent of that invoked for Galactic binaries, with the accretion disk becoming more prominent as the luminosity increases. However, although the disk temperature and luminosity are well correlated across all time scales currently probed, the observed luminosity follows $L \propto T^{1.70 \pm 0.17}$, flatter than expected for simple blackbody radiation. The spectral variability displayed here is highly reminiscent of that observed from known Galactic black hole binaries (BHBs) at high luminosities. This comparison implies a black hole mass of $\sim 90 M_{\odot}$ for Circinus ULX5. However, given the diverse behavior observed from Galactic BHB accretion disks, this mass estimate is still uncertain. Finally, the limits placed on any undetected iron absorption features with the 2013 data set imply that we are not viewing the central regions of Circinus ULX5 through any extreme super-Eddington outflow.
\end{abstract}

Key words: black hole physics - X-rays: binaries - X-rays: individual (Circinus ULX5)

Online-only material: color figures

\section{INTRODUCTION}

The origin of the extreme luminosities displayed by ultraluminous X-ray sources (ULXs; $L_{\mathrm{X}} \gtrsim 10^{39} \mathrm{erg} \mathrm{s}^{-1}$ ) may relate to exotic super-Eddington modes of accretion (e.g., Poutanen et al. 2007, Finke \& Böttcher 2007), or alternatively to the presence of black holes larger than those typically found in Galactic black hole binary systems (BHBs; $M_{\mathrm{BH}} \sim 10 M_{\odot}$ ), potentially the long sought intermediate mass black holes (IMBHs: $10^{2} \lesssim M_{\mathrm{BH}} \lesssim$ $10^{5} M_{\odot}$; e.g., Miller et al. 2004; Strohmayer 2009). For recent reviews see Roberts (2007) and Feng \& Soria (2011).

The majority of ULXs only modestly exceed $10^{39} \mathrm{erg} \mathrm{s}^{-1}$, and therefore likely represent a natural extension of the diskdominated high-Eddington thermal states displayed by Galactic BHBs (e.g., Kajava \& Poutanen 2009; Middleton et al. 2013). However, a much smaller fraction of the ULX population have been observed to exceed $10^{40} \mathrm{erg} \mathrm{s}^{-1}$ in X-rays (Walton et al. 2011b; Jonker et al. 2012; Sutton et al. 2012), and to reach as high as $\sim 10^{42} \mathrm{erg} \mathrm{s}^{-1}$ (Farrell et al. 2009) in the most luminous case known to date. These more luminous sources, apparently radiating in excess of ten times the Eddington limit for a $10 M_{\odot}$ black hole, remain among the best known candidates to host massive black holes.

Here we report on an extremely bright and highly variable ULX in the outskirts of the Circinus galaxy $(z=0.001448$, $D \sim 4$ Mpc; Freeman et al. 1977; Koribalski et al. 2004), hereafter Circinus ULX5 (there are up to four other known/claimed ULX candidates closer to the Circinus nucleus, see e.g., Bauer et al. 2001; Swartz et al. 2004; Liu \& Mirabel 2005; Ptak et al. 2006). Circinus ULX5 was serendipitously detected in a high luminosity state by the recently launched Nuclear Spectroscopic Telescope Array (NuSTAR) on 2013 January 25, and we subsequently performed follow-up target-of-opportunity (ToO) observations with both XMM-Newton and NuSTAR (Harrison et al. 


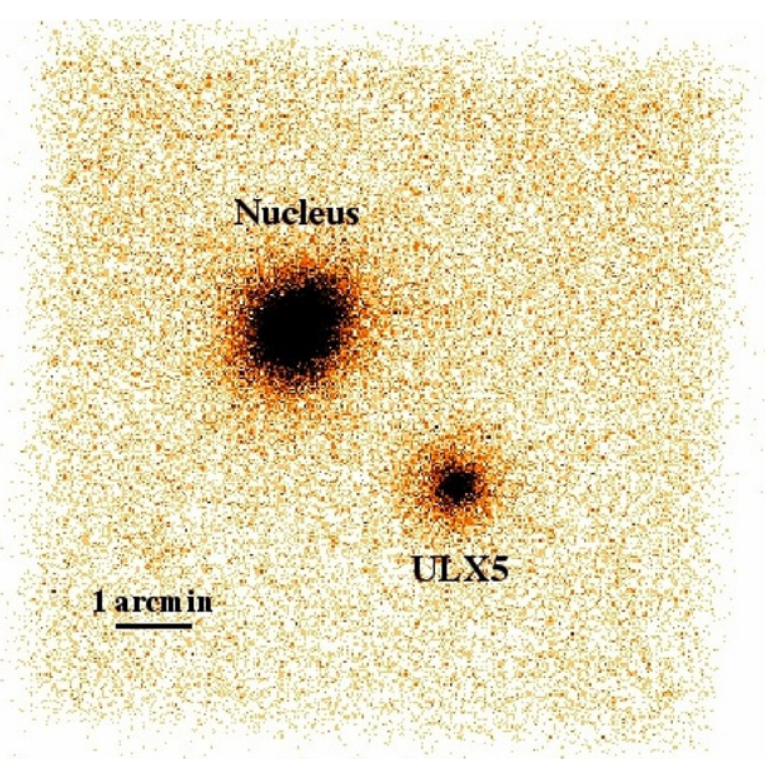

Figure 1. NUSTAR FPMA image of the Circinus field. The Circinus nucleus and Circinus ULX5, separated by $\sim 4^{\prime}$, are both highlighted.

(A color version of this figure is available in the online journal.)

2013). In spite of its luminosity, Circinus ULX5 has received little attention to date, owing in large part to its fairly substantial separation from the galaxy center (Circinus ULX5 formally falls outside the D25 isophote for Circinus). The only mention of the source is in the Winter et al. (2006) ULX catalogue. ${ }^{14}$ In Section 2 we detail our data reduction procedure for the various data sets considered and in Sections 3-5 we describe our multiepoch spectral and temporal analysis of this remarkable source. Key results are discussed in Section 6 and we summarize our conclusions in Section 7. Throughout this work, we will assume Circinus ULX5 is indeed associated with the Circinus galaxy. Possible alternative scenarios are discussed in Section 6.1, but we consider them highly unlikely.

\section{DATA REDUCTION}

Here we outline our data reduction procedure for the X-ray observations considered in this work, beginning with the new XMM-Newton and NuSTAR data sets obtained in early 2013.

\subsection{Observations}

\subsubsection{NUSTAR}

NuSTAR (Harrison et al. 2013) performed four observations of the Circinus field throughout 2013 in late January and early February. The first observation, in which the bright ULX was serendipitously detected, was optimized to observe the galaxy's nucleus, but the three subsequent follow-up observations were all optimized to observe the ULX, which is offset from the nucleus by $\sim 4^{\prime}$ (see Figure 1): R.A. $=14^{\mathrm{h}} 12^{\mathrm{m}} 39^{\mathrm{s}}$, decl. $=$ $-65^{\circ} 23^{\prime} 34^{\prime \prime}$. The data have been reduced using the standard pipeline, part of the NUSTAR Data Analysis Software v0.11.1 (NUSTARDAS, part of the standard HEASOFT distribution as of version 14), and instrumental responses from $N u S T A R$ caldb $^{15}$ v20130509 are used throughout. As discussed in Risaliti

\footnotetext{
14 Note that in Winter et al. (2006), the source studied here is referred to as Circinus XMM2.

15 The NUSTAR calibration database is available through HEASARC: http://heasarc.gsfc.nasa.gov/docs/heasarc/caldb/nustar
}

et al. (2013), these responses have been empirically corrected such that the Crab nebula gives a power law spectrum with a photon index of $\Gamma_{\text {Crab }}=2.1$. Full details are provided in $\mathrm{K}$. K. Madsen et al. (2013, in preparation), but the calibration has also been successfully tested against other power-law-like sources, including the pulsar wind nebula G 21.5-0.9 and the blazar PKS 2155, which are frequently used to assess the calibration of X-ray missions (Tsujimoto et al. 2011; Ishida et al. 2011). The unfiltered event files have been cleaned with the standard depth correction, which significantly reduces the internal background at high energies, and South Atlantic Anomaly passages have been removed. Source products were obtained from circular regions of $\sim 70^{\prime \prime}$ in radius for the observations with the ULX on the optical axis, and $\sim 50^{\prime \prime}$ in radius for the initial detection to avoid the edge of the detector. Background was estimated from a blank area of the same detector, free from contaminating point sources. Spectra and lightcurves were extracted from the cleaned event files using XSELECT for both focal plane modules (FPMA and FPMB). Finally, the spectra were grouped with GRPPHA such that each spectral bin contains at least 50 counts. Although NuSTAR operates over the 3-79 keV energy range, Circinus ULX5 is only reliably detected up to $\sim 30 \mathrm{keV}$.

\subsubsection{XMM-Newton}

After the initial serendipitous NuSTAR detection, we also triggered a $\sim 50 \mathrm{ks}$ ToO observation with XMM-Newton (Jansen et al. 2001) in order to provide complimentary soft X-ray coverage for our targeted NuSTAR follow-up observations (performed simultaneously with NuSTAR ObsID 30002038004). Data reduction was carried out with the XMM-Newton Science Analysis System (SASv12.0.1) largely according to the standard prescription provided in the online guide. ${ }^{16}$ The observation data files were processed using EPCHAIN and EMCHAIN to produce calibrated event lists for the EPIC-pn (Strüder et al. 2001) and EPIC-MOS (Turner et al. 2001) detectors, respectively. For each detector, source products were extracted from a circular region of $\sim 40^{\prime \prime}$ in radius, and background was estimated from an area of the same CCD, free of contaminating point sources. Lightcurves and spectra were generated with XMMSELECT, selecting only single and double events (single to quadruple events) for EPIC-pn (EPIC-MOS), excluding periods of high background flares (occurring predominantly at the end of the observation). The redistribution matrices and auxiliary response files were generated with RMFGEN and ARFGEN, while lightcurves were corrected for the background count rate using EPICLCCORR. After performing the data reduction separately for each of the MOS CCDs and confirming their consistency, the spectra were combined using the FTOOL ADDASCASPEC. Finally, spectra were re-binned to have a minimum of 50 counts in each energy bin, and analyzed across the full $0.3-10.0 \mathrm{keV}$ energy range.

\subsection{Archival Data}

Prompted by the new data obtained in 2013, we also searched the X-ray archive for observations of the Circinus field, in order to investigate potential long term variability. A summary of all the X-ray observations considered in this work is given in Table 1 . The additional archival observations were almost all targeted at the Circinus nucleus or the nearby supernova SN1996cr

\footnotetext{
16 http://xmm.esac.esa.int/
} 
Table 1

Details of the X-Ray Observations Considered in This Work, Ordered Chronologically

\begin{tabular}{|c|c|c|c|c|c|}
\hline Mission & ObsID & Date & Target & $\begin{array}{c}\text { Good Exposure } \\
(\mathrm{ks})\end{array}$ & $\begin{array}{l}\text { ULX5 3-10 keV Flux } \\
\left(10^{-12} \mathrm{erg} \mathrm{cm}^{-2} \mathrm{~s}^{-1}\right)\end{array}$ \\
\hline XMM-Newton & 0111240101 & 2001 Aug 6 & Nucleus & $105 / 110 / \mathrm{-}^{\mathrm{a}}$ & $1.09 \pm 0.04$ \\
\hline Suzaku & 701036010 & 2006 Jul 21 & Nucleus & 110 & $1.55 \pm 0.04$ \\
\hline Swift & 00035876001 & 2007 Mar 23 & Field & 7.5 & $0.8_{-0.3}^{+0.1}$ \\
\hline Swift & 00037273001 & 2008 May 18 & Field & 6 & $1.0_{-0.4}^{+0.3}$ \\
\hline Chandra & 10873 & 2009 Mar 1 & SN1996cr & 18 & $5.0 \pm 0.5$ \\
\hline Chandra & 10850 & 2009 Mar 3 & SN1996cr & 14 & $4.8_{-0.5}^{+0.4}$ \\
\hline Chandra & 10872 & 2009 Mar 4 & SN1996cr & 17 & $1.0_{-0.1}^{+0.2}$ \\
\hline Swift & 00090260001 & 2009 Nov 15 & Field & 5 & $2.3_{-0.6}^{+1.0}$ \\
\hline Swift & 00037273004 & 2012 Nov 11 & Field & 3.5 & $1.0_{-0.5}^{+0.7}$ \\
\hline NUSTAR & 60002039002 & 2013 Jan 25 & Nucleus & 55 & $4.2 \pm 0.2$ \\
\hline Swift & 00032699001 & 2013 Jan 31 & Field & 3.5 & $5.0_{-1.0}^{+1.3}$ \\
\hline NuSTAR & 30002038002 & 2013 Feb 2 & ULX5 & 18 & $4.2 \pm 0.2$ \\
\hline XMM-Newton & 0701981001 & 2013 Feb 3 & ULX5 & $37 / 47 / 47^{\mathrm{a}}$ & (as below) \\
\hline NuSTAR & 30002038004 & 2013 Feb 3 & ULX5 & 40 & $4.93 \pm 0.12$ \\
\hline NuSTAR & 30002038006 & 2013 Feb 5 & ULX5 & 36 & $3.7 \pm 0.1$ \\
\hline
\end{tabular}

Notes.

${ }^{a}$ XMM-Newton exposures are listed for the EPIC-pn/MOS1/MOS2 detectors.

b Observed fluxes are computed in Section 3 for the higher $\mathrm{S} / \mathrm{N}$ observations, utilizing the DISKBB+SIMPL model, and in 5.1 for the shorter snapshot observations, utilizing a simpler cutoff power law model.

(Bauer et al. 2008), but Circinus ULX5 is serendipitously included in the field of view, albeit substantially off-axis, in each case.

\subsubsection{XMM-Newton}

In addition to the recent ToO we obtained, XMM-Newton also observed Circinus in 2001 August. This observation was largely reduced in the same manner as the ToO described above (Section 2.1.2). However, in this case the target was the Circinus nucleus, and the ULX in question unfortunately fell very close to a chip gap in the EPIC-pn detector. Furthermore, the MOS2 detector was operated in partial window mode, and the target fell outside the operational region of the detector. In this case, we use an elliptical source region for EPIC-pn, offset slightly from the source position in order to include as many counts as possible without the region covering the chip gap. The shape of the EPIC-pn spectrum obtained is consistent with that obtained with MOS1, however we do find that the flux normalization is not consistent between the detectors. We therefore take the MOS1 detector as the true flux indicator for this observation, given the non-standard reduction necessary for EPIC-pn. In this case, as the average countrate is much lower than the more recent $X M M$-Newton observation, owing to the combined effect of a large off-axis angle and a lower source flux (see Section 3.3 and Table 1), we instead rebin to a minimum of 25 counts per bin.

\subsubsection{Suzaku}

The Circinus field was also observed by Suzaku (Mitsuda et al. 2007) in 2006 July. Owing to the obvious, dominant contribution from the nucleus, we do not consider the HXD detectors in this work, and focus instead on the data obtained with the XIS CCDs. Following the recommendation in the Suzaku Data Reduction Guide, ${ }^{17}$ we reprocessed the unfiltered event files for each of the operational XIS detectors (XIS0, 1, 2 and 3; Koyama et al. 2007) and editing modes $(3 \times 3$ and $5 \times 5)$ using the latest HEASOFT software package (v6.13). Cleaned event

\footnotetext{
17 http://heasarc.gsfc.nasa.gov/docs/suzaku/analysis/
}

files were generated by re-running the Suzaku pipeline with the latest calibration, as well as the associated screening criteria files. Source products were extracted from a circular region of $\sim 85^{\prime \prime}$ in radius, in order to avoid contamination from a further faint source nearby (separated by $\sim 105^{\prime \prime}$ ). The background was extracted from regions free of any obvious contaminating point sources, but close to the source region. Spectra and lightcurves were extracted from the cleaned event files with XSELECT, and responses were generated for each detector using the XISRESP script with a medium resolution. The spectra and response files for the front-illuminated detectors (XIS0, 2 and 3) were combined using the FTOOL ADDASCASPEC, after confirming their consistency. Finally, we again grouped the spectra to have a minimum of 50 counts per energy bin. In this work, we analyze the XIS data over the $0.5-10.0 \mathrm{keV}$ energy range.

\subsubsection{Chandra}

Although the Circinus field has also frequently been observed by the Chandra X-ray observatory (Weisskopf et al. 2002), Circinus ULX5 only fell in the field of view for three of these pointings (see Table 1). For each of the three observations, the instrument was operated in the Timed Event mode, and we extracted spectra from the ACIS-S detector (Garmire et al. 2003) using the standard pipeline CIAO v4.5. In all observations Circinus ULX5 is detected close to the edge of the field of view, while the observatory pointed at SN1996cr. At these large, offaxis angles the Chandra PSF is clearly elongated, so we used an elliptical extraction region with major and minor axes of $5.5 \times 2^{\prime \prime} .7$ to gather all source photons. The background was extracted from two large circular regions above and below the HETG diffraction pattern, free from any other contaminating sources. The Chandra spectra were rebinned to a signal-tonoise ratio $(\mathrm{S} / \mathrm{N})$ of 2 , and modelled over the full $0.3-9.0 \mathrm{keV}$ energy range.

\subsubsection{Swift Snapshots}

Finally, Swift (Gehrels et al. 2004) has also sporadically taken snapshot observations of the Circinus field. We searched the 
Swift archive for pointed observations with at least $1 \mathrm{ks}$ duration, such that reasonable flux estimates might be obtained, and found five observations that met our criteria. Cleaned event files were generated in the standard manner with XRTPIPELINE, and spectral products were extracted with XSELECT. Source spectra were taken from circular regions of radius $\sim 30^{\prime \prime}$, and background spectra from larger, adjacent regions free of contaminating point sources. Ancillary responses were generated with XRTMKARF, and we use the latest redistribution matrices available in the Swift calibration database (v13). The Swift spectra were only grouped to have at least 5 counts per spectral bin, such that even the observations with the lowest S/N (ObsIDs 00037273001 and 00037273004 ) had at least ten spectral bins across the $0.3-10.0 \mathrm{keV}$ energy range.

\section{SPECTRAL ANALYSIS}

The majority of our spectral analysis focuses on the long, higher S/N observations of the ULX, i.e., the joint 2013 XMMNewton and NuSTAR data set, the 2006 Suzaku data, and the 2001 XMM-Newton data. Throughout this work, spectral modelling is performed with XSPEC v12.8.0 (Arnaud 1996), and, unless stated otherwise, quoted uncertainties on spectral parameters are the 90 percent confidence limits for a single parameter of interest. Unless stated otherwise, spectral fitting is performed through $\chi^{2}$ minimization. Neutral absorption is treated with TBNEW, ${ }^{18}$ the latest version of the TBABS absorption code (Wilms et al. 2000), with the appropriate solar abundances. Unless stated otherwise, all models include Galactic absorption with a column of $N_{\mathrm{H} ; \mathrm{Gal}}=5.58 \times 10^{21} \mathrm{~cm}^{-2}$ (Kalberla et al. 2005). In the following, data from a variety of $\mathrm{X}$-ray missions are utilized, many of which operate multiple detectors simultaneously (e.g., EPIC-pn and EPIC-MOS aboard $X M M$-Newton). In these cases, the data from the different detectors are modelled simultaneously, with all parameters tied between the spectra. However, we attempt to account for any residual internal cross-calibration uncertainties between the detectors by including a variable multiplicative crossnormalization constant. This value is almost always found to be within $\sim 5$ percent of unity for all such missions, with the only exception being the 2001 XMM-Newton data, owing to the unfortunate position of the source on the EPIC-pn detector as previously discussed (see Section 2.2.1).

\subsection{NuSTAR and XMM-Newton in 2013}

\subsubsection{Cross-calibration}

We begin our analysis with the recent broadband $X M M-N e w t o n+N u S T A R$ spectrum. When modelling this joint data set, we treat possible issues with flux cross-calibration between NuSTAR and XMM-Newton in the same way as we do cross-calibration uncertainties between different detectors within a single mission (see above). The individual $N u S T A R$ and $X M M$-Newton data sets have substantial spectral overlap, both covering the 3-10 keV energy range, from which crossnormalization constants can easily be constrained. In order to demonstrate the spectral consistency between XMM-Newton and NUSTAR, we initially focus on this energy range.

Applying a simple power law model, modified by Galactic absorption results in a poor fit, with $\chi_{v}^{2}=796 / 565$ and clear curvature present in the residuals for both the XMM-Newton and NuSTAR data (Figure 2, upper panel). Inspection of the

\footnotetext{
18 http://pulsar.sternwarte.uni-erlangen.de/wilms/research/tbabs
}

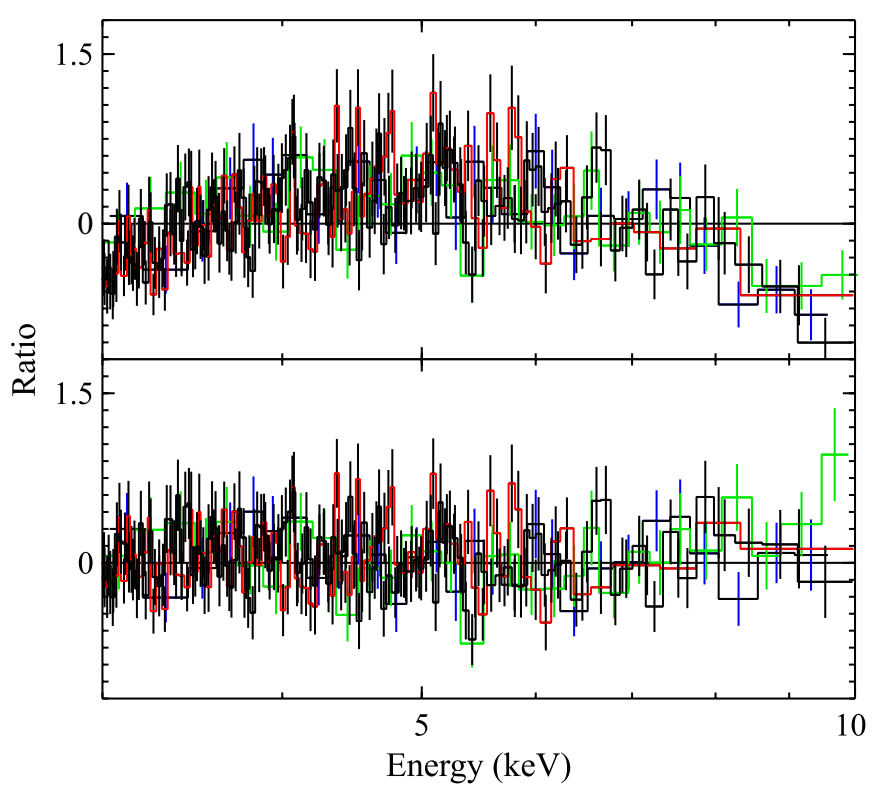

Figure 2. Data/model ratios for the simultaneous $X M M$-Newton (black: EPIC-pn, red: EPIC-MOS) and NuSTAR (green: FPMA, blue: FPMB) data sets, modelled with both a power law continuum modified by Galactic absorption (top panel) and an unabsorbed disk component (bottom panel). The XMMNewton and NuSTAR data sets display clear and consistent curvature across their common energy range (3-10 keV).

(A color version of this figure is available in the online journal.)

full $0.3-10.0 \mathrm{keV}$ XMM-Newton data suggests the overall neutral column is most likely in excess of the Galactic column, closer to $N_{\mathrm{H} \text {; tot }} \sim 10^{22}$ atom $\mathrm{cm}^{-2}$, but even allowing for a column of this order does not fully remove the curvature in the 3-10 keV bandpass (a total column of $N_{\mathrm{H} \text {; tot }} \sim 10^{22}$ atom $\mathrm{cm}^{-2}$ still has a very limited effect above $3 \mathrm{keV}$ ). We therefore conclude that the $3-10 \mathrm{keV}$ continuum of Circinus ULX5 is intrinsically curved, similar to other bright ULXs (Stobbart et al. 2006; Gladstone et al. 2009; Walton et al. 2011a). If we instead model the $3-10 \mathrm{keV}$ data with a curved continuum, simply parameterizing the data with an unabsorbed DISKBB component (Mitsuda et al. 1984), we obtain an excellent fit with $\chi_{v}^{2}=563 / 564$ (Figure 2, lower panel). Allowing the $X M M-N e w t o n$ and NuSTAR temperatures to vary independently does not improve the fit at all $\left(\chi_{v}^{2}=563 / 563\right)$, and we obtain $T_{\text {XMM }}=2.01 \pm 0.04 \mathrm{keV}$ and $T_{\text {NUSTAR }}=2.04 \pm 0.06 \mathrm{keV}$. Clearly the 3-10 keV spectra obtained with XMM-Newton and $N U S T A R$ are fully consistent. Furthermore, the NuSTAR and EPIC-MOS fluxes agree to within $\sim 15 \%$.

\subsubsection{Continuum Modelling}

We now consider the full $0.3-30.0 \mathrm{keV}$ broadband spectrum, and model the XMM-Newton and NuSTAR data simultaneously. In addition, we now (and hereafter) formally include both Galactic absorption and intrinsic neutral absorption (at the redshift of Circinus), the latter being free to vary. Naturally, the simple power law continuum continues to provide a very poor fit $\left(\chi_{v}^{2}=3388 / 1119\right)$. However, applying the simple accretion disk continuum also results in a fairly poor fit $\left(\chi_{v}^{2}=1287 / 1119\right)$, and clear divergence between the data and the model can be seen in the residuals at high energies ( $\gtrsim 10 \mathrm{keV}$; see Figure 3 ), where the Wien tail of the DISKBB model falls away far faster than the data.

Initially, we attempt to model this additional high energy emission with a power-law-like Comptonised component, 


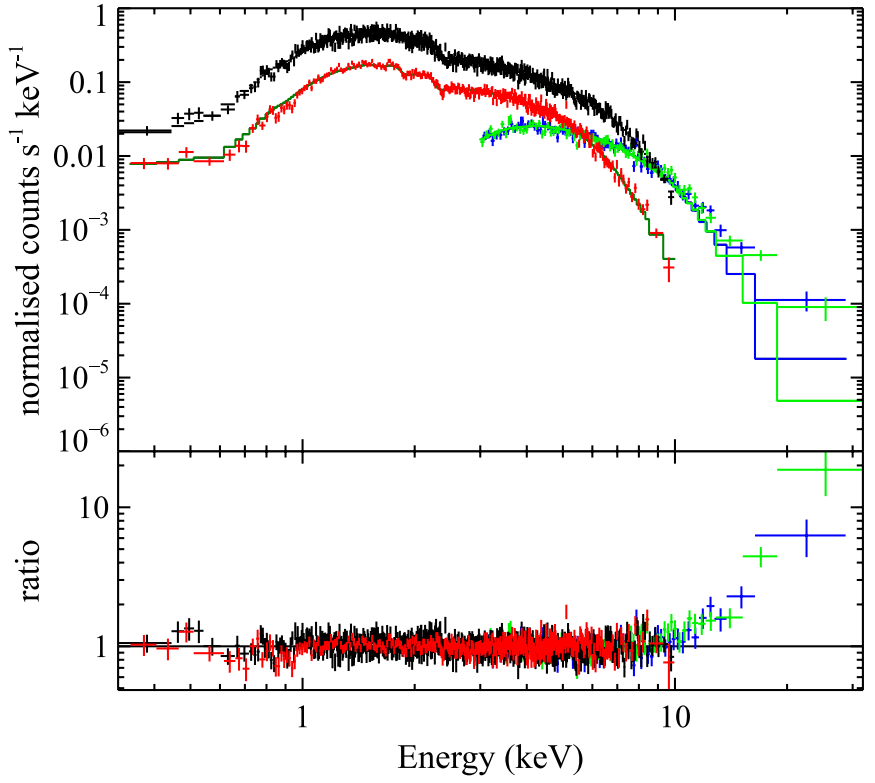

Figure 3. Broadband $0.3-30.0 \mathrm{keV}$ spectrum of Circinus ULX5 modelled with a simple DISKBB accretion disk model. This model results in a clear excess in the NUSTAR data above $10 \mathrm{keV}$.

(A color version of this figure is available in the online journal.)

applying the SIMPL convolution model (Steiner et al. 2009), which "scatters" some fraction of an input seed photon distribution into a high energy power law tail, to the DISKBB continuum. We use this model rather than a basic power law component in order to ensure that the power law does not extrapolate to arbitrarily low energies, which is potentially important given the high temperature of the disk $(\sim 2 \mathrm{keV})$. SIMPL has three parameters, the photon index of the high energy tail, the fraction of the seed flux scattered into the high energy tail $\left(f_{\text {scat }}\right)$, and a flag determining whether to allow for both Compton upand down-scattering, or just the former. For simplicity, we only allow for up-scattering of the seed photon spectrum, although the results obtained are not sensitive to this assumption. The addition of this component significantly improves the fit, with $\chi_{v}^{2}=1137 / 1117$, i.e., an improvement of $\Delta \chi^{2}=150$ for two additional free parameters, and resolves the excess at high energies. The parameter values obtained are quoted in Table 2 . However, a number of parameters are found to be degenerate with one another; this is particularly the case for the parameters that determine the high energy spectrum (see Figure 4). Although fairly poorly constrained, owing to these degeneracies, the photon index obtained is very steep, $\Gamma=4.0_{-0.8}^{+0.4}$.

The full $0.3-30.0 \mathrm{keV}$ observed flux from Circinus ULX5 during this epoch is $(8.5 \pm 0.3) \times 10^{-12} \mathrm{erg} \mathrm{cm}^{-2} \mathrm{~s}^{-1}$. Owing to the steep high energy spectrum, this is mostly dominated by the emission below $10 \mathrm{keV}$, which contributes 85 per cent of the total $0.3-30.0 \mathrm{keV}$ flux. At the distance of Circinus ( $D \sim 4 \mathrm{Mpc}$; Freeman et al. 1977; Koribalski et al. 2004), the broadband flux corresponds to an extreme luminosity of $L_{0.3-30.0}=(1.63 \pm 0.06) \times 10^{40} \mathrm{erg} \mathrm{s}^{-1}$, assuming isotropic emission, placing Circinus ULX5 amongst the most luminous ULXs known to date, even before absorption corrections are considered. Although the exact correction is somewhat model dependent, for the DISKBB+SIMPL combination, the intrinsic $0.3-30.0 \mathrm{keV}$ luminosity inferred is $\sim 2 \times 10^{40} \mathrm{erg} \mathrm{s}^{-1}$, a further $\sim 20$ percent larger than the observed luminosity.

Table 2

Best Fit Parameters Obtained for the Variety of Continuum Models Applied to the High S/N Data Available for Circinus ULX5

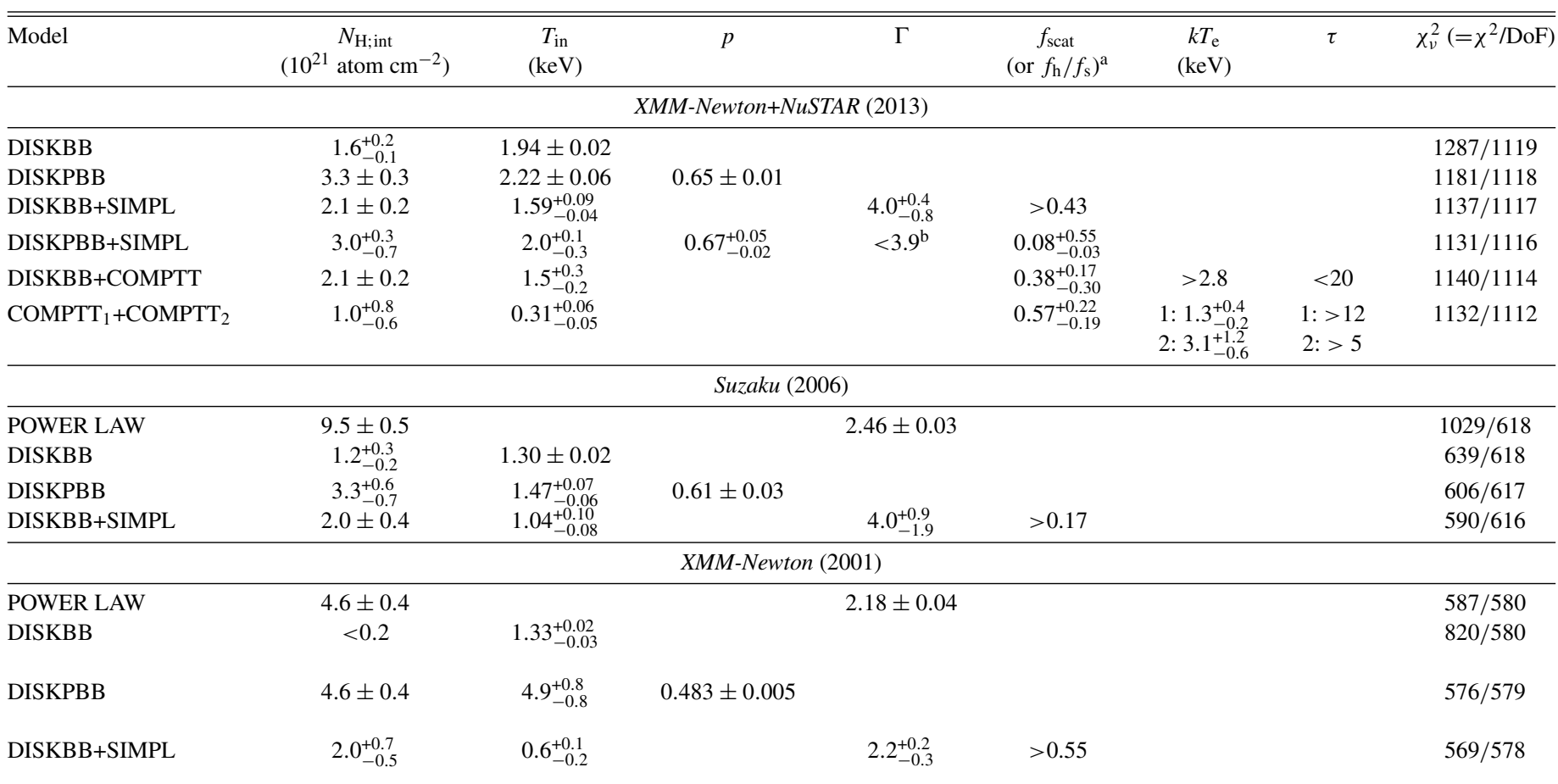

Notes.

${ }^{a}$ For models that do not include SIMPL, hard and soft component fluxes $\left(f_{\mathrm{h}}\right.$ and $f_{\mathrm{s}}$ respectively) are calculated extrapolating the model components over the energy range $0.01-100 \mathrm{keV}$. In the two COMPTT model, the higher temperature component is the harder of the two.

${ }^{\mathrm{b}}$ SIMPL does not allow for photon indices below $\sim 1.1$. 

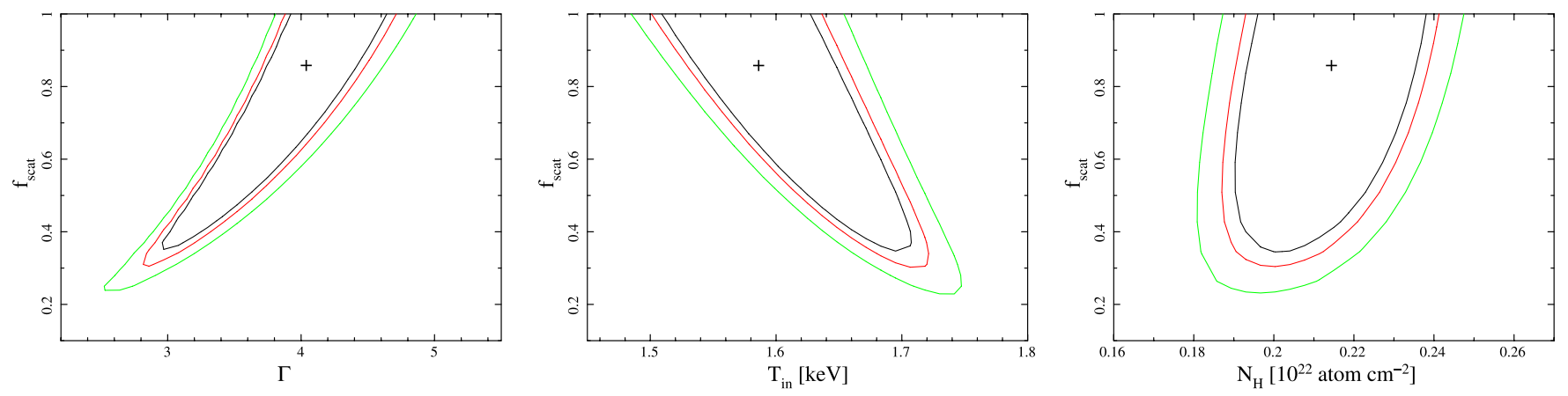

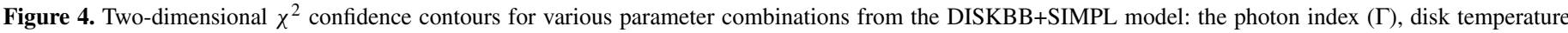

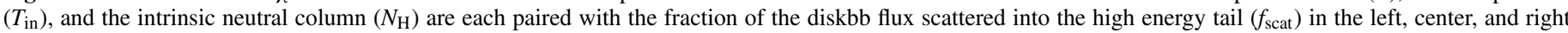

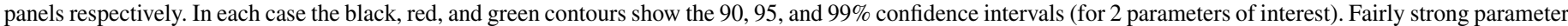
degeneracies are observed in some cases.

(A color version of this figure is available in the online journal.)

Given the steep nature of the high energy spectrum, it is not clear that the hard excess can be considered as similar to the hard excesses frequently seen in active galactic nuclei (AGNs; e.g., Walton et al. 2010, 2013b; Nardini et al. 2011; Risaliti et al. 2013; Rivers et al. 2013) and BHBs (e.g., Zdziarski et al. 2002; Corongiu et al. 2003; Reis et al. 2010), which are best associated with Compton reflection (and if phenomenologically modelled with a power law would generally give $\Gamma \ll 2$ ). It has recently been suggested that the combination of iron emission and absorption in a relativistically smeared reflection spectrum from the inner regions of the accretion disc might be able to explain the curvature observed below $10 \mathrm{keV}$ in bright ULXs (Caballero-García \& Fabian 2010). This would then allow the intrinsic high energy emission to have a powerlaw-like form, as is typical for sub-Eddington coronal emission. In general, this interpretation required high iron abundances and strong relativistic broadening in order to reproduce the smooth 3-10 keV curvature (Caballero-García \& Fabian 2010; Walton et al. 2011a). However, while such a model, consisting of a power-law-like corona and a smeared reflection component (modelled with a combination of the REFLIONX reflection code, Ross \& Fabian 2005, and the RELCONV relativistic kernel, Dauser et al. 2010) does provide an adequate fit to the $X M M$-Newton data alone $\left(\chi_{v}^{2}=1018 / 950\right)$, when fit to the broadband spectrum, the Compton reflection hump at $\sim 20 \mathrm{keV}$ is in significant excess of the observed NuSTAR data, as is clear from Figure 5 (see also Walton et al. 2011a). The resulting broadband fit is rather poor $\left(\chi_{v}^{2}=1623 / 1111\right)$.

In this case, however, there is formally an alternative solution using this model combination that provides an acceptable fit to the broadband spectrum $\left(\chi_{v}^{2}=1145 / 1113\right)$, although it is rather different than previous applications to ULXs. Rather than model the curvature with iron emission/absorption, this fit instead attempts to remove all iron features, and requires the lowest iron abundance allowed by the model. ${ }^{19}$ Without any iron absorption at $\sim 7 \mathrm{keV}$, the peak of the Compton hump shifts to lower energies. In addition, the spin obtained is very high, the radial emissivity index is maximized, the disk is required to be face on, and its ionization state is minimized. This combination serves to further reduce the energy of the peak of the Compton hump, to the extent that the $3-10 \mathrm{keV}$ curvature is actually modelled by this aspect of the reflected emission. In fact, in this

\footnotetext{
19 The REFLIONX grid utilized is calculated for photon indices in the range $\Gamma=1.4-3.3$, ionization states in the range $\log \xi=0-4$, and iron abundances in the range $0.1-10.0$.
}

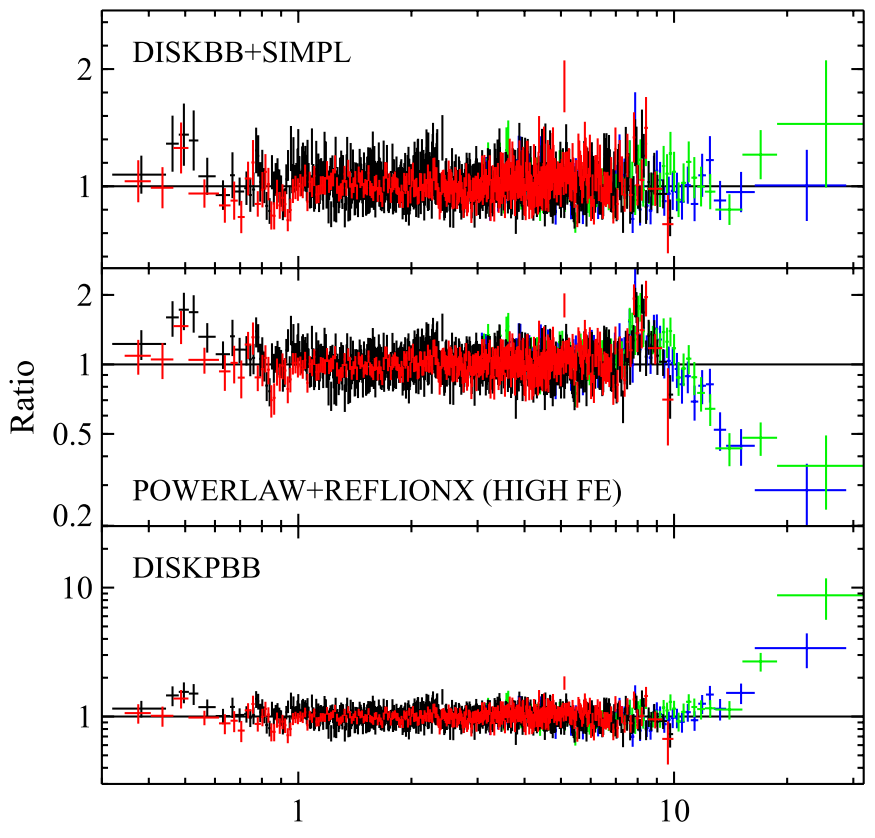

Figure 5. Data/model ratios for a selection of the models applied to the combined XMM-Newton+NuSTAR data set for Circinus ULX5 (see text for details). Top panel: the DISKBB+SIMPL combination, which provides an excellent fit to the broadband spectrum. Middle panel: the relativistic disk reflection model in which the $3-10 \mathrm{keV}$ curvature is modelled as blurred iron emission/absorption (the high iron abundance fit), which severely over predicts the high energy NUSTAR data. Bottom panel: the DISKPBB model, which still under predicts the high energy data, similar to the simpler DISKBB model.

(A color version of this figure is available in the online journal.)

extreme corner of parameter space, the reflection component is smeared and shifted to such an extent that, when absorbed by a substantial neutral column, it takes on the appearance of a hot thermal-like spectrum with a steep power law tail. All the features typically associated with reflected emission are essentially removed, which we interpret as further evidence that the spectrum of Circinus ULX5 is not well modelled with traditional disc reflection. Therefore, although statistically acceptable, we consider this to be a very unsatisfying solution. If reflection is a relevant process for ULXs, the picture must be more complex than the standard thin disk-corona accretion geometry. Owing to the complexity of the model, we do not include the obtained results in Table 2. 
If ULXs do represent a population of sources accreting at very high or super-Eddington rates, the expected emission from the accretion disc may in fact be substantially different from the simple Shakura \& Sunyaev (1973) thin disc profile assumed in the DISKBB model. As the accretion rate increases toward substantial Eddington fractions, the scale height of the disc is expected to increase, and advection becomes an increasingly important process (Abramowicz et al. 1988), resulting in shallower radial temperature profiles and hence giving the appearance of a broader, less peaked emission profile from the disc. In order to investigate whether a broader disc profile could potentially account for the additional hard emission relative to the simple DISKBB profile, we attempted to model the broadband spectrum with a DISKPBB model, which includes the index of the radial temperature profile $(p)$ as an additional free parameter (Mineshige et al. 1994). This offers a substantial improvement over the pure DISKBB model, with $\chi_{v}^{2}=1181 / 1118$, and the radial temperature profile obtained is indeed shallower than expected for a thin disc (i.e., $p<0.75$ ). However, as shown in Figure 5 we again see an excess of emission over the DISKPBB model, although it is slightly weaker than in the DISKBB case, owing to the disc emission being able to extend to higher energies while still reproducing the observed curvature in the 3-10 keV bandpass. An additional component is still required. In fact, this is the case for any model invoked to explain the curvature below $10 \mathrm{keV}$ that falls away above $10 \mathrm{keV}$ with a thermal Wien spectrum, including more detailed accretion disc models (e.g., KERRBB; Li et al. 2005) and optically thick Comptonisation by cool ( $2 \mathrm{keV}$ ) electrons (e.g., Gladstone et al. 2009).

Adding a Comptonised component (SIMPL) to the DISKPBB model again provides a clear improvement to the fit, with $\chi_{v}^{2}=$ $1131 / 1116$, i.e., an improvement of $\Delta \chi^{2}=50$ for two additional free parameters over the pure DISKPBB model. However, the improvement over the DISKBB+SIMPL combination is very marginal, $\Delta \chi^{2}=6$ for one additional free parameter, and the additional model complexity serves to further exacerbate the parameter degeneracies already present with the DISKBB+SIMPL model (Figure 4). The best fit radial temperature profile for the disc is only marginally constrained to be shallower than expected for the thin disc case $\left(p=0.67_{-0.02}^{+0.05}\right)$, and in this instance we find that the photon index is only loosely constrained at all $(\Gamma<3.9)$.

For completeness, we also fit the DISKBB+COMPTT combination, frequently used to parameterize the spectra from bright ULXs (e.g., Gladstone et al. 2009; Middleton et al. 2011; Walton et al. 2011a, 2012a), which allows for a variable electron temperature for the thermal Comptonisation (Titarchuk 1994). Unsurprisingly, this also provides an excellent fit $\left(\chi_{v}^{2}=\right.$ $1140 / 1114$ ), although, again, the additional model complexity does not offer any substantial improvement. The same model combination with the electron temperature fixed at $500 \mathrm{keV}$, such that in the NUSTAR band the COMPTT component is largely power-law-like, provides an equally good fit $\left(\chi_{v}^{2}=1142 / 1115\right)$. There is again substantial degeneracy between the various physical parameters, but despite this, there is a marked difference between the fit parameters for Circinus ULX5 and those obtained for other bright $\left(L_{\mathrm{X}} \sim 10^{40} \mathrm{erg} \mathrm{s}^{-1}\right)$ ULXs. Here, it is the DISKBB component that primarily produces the $3-10 \mathrm{keV}$ curvature, while in previous work this curvature is accounted for by the COMPTT component, resulting in cool, optically thick electron distributions being inferred. The DISKBB component instead usually accounts for the additional soft emission seen below $\sim 1 \mathrm{keV}$ in bright ULXs with less absorption (e.g., Miller et al. 2003, 2004, 2013). Unfortunately, owing to the fairly substantial total absorbing column toward Circinus ULX5, we are not highly sensitive to the presence of any such emission. In this case, the COMPTT component instead accounts for the excess emission observed above $10 \mathrm{keV}$. As such, the Comptonisation parameters are not well constrained (see Table 2). Nevertheless, the electron temperature obtained is still higher than typical results from analyses limited to below $10 \mathrm{keV}$, which find $T_{\mathrm{e}} \sim 2 \mathrm{keV}$, or less (e.g., Gladstone et al. 2009; Walton et al. 2011a).

Finally, we also consider a dual Comptonisation model for Circinus ULX5, employing two COMPTT continuum components. Such dual-coronae have been proposed for Galactic BHBs in some cases (e.g., Makishima et al. 2008), but this is the first time such a model has been applied to a ULX. For simplicity, the seed photon temperatures for the two components are linked throughout most of our analysis. Initially, following Makishima et al. (2008), we attempted to fit the data with a common electron temperature for each COMPTT component, with the two merely having differing optical depths. However, this resulted in a relatively poor fit $\left(\chi_{v}^{2}=1199 / 1113\right)$, with the model failing to correctly account for the high energy emission, similar to Figure 3. Two different electron temperatures are strongly required, as one of the components is required to model the 3-10 keV curvature, while the other needs to extend to higher energies in order to model the residual high energy excess. Allowing for two different electron temperatures, an excellent fit is obtained $\left(\chi_{v}^{2}=1132 / 1112\right)$. With this configuration, both electron distributions are found to be optically thick (see Table 2), although this is no longer the case if the two components are allowed to have different seed photon temperatures, in which case the parameters of the COMPTT component that accounts for the high energy excess are only poorly constrained, as before.

\subsubsection{The Iron K Region}

The combined XMM-Newton+NuSTAR data set has sufficient photon statistics at high energies to warrant an investigation of the iron $\mathrm{K}$ region $(6-7 \mathrm{keV})$. Due to their typically moderate fluxes and their frequently soft spectra, ULX data sets sensitive in the iron $\mathrm{K}$ energy range are naturally rare. For bright $\left(L_{\mathrm{X}}>10^{40} \mathrm{erg} \mathrm{s}^{-1}\right)$, isolated ULXs, such studies have to date been limited to Holmberg IX X-1 and NGC 1313 X-1 (Walton et al. 2012a, 2013a).

To search for atomic features here, we follow the same approach undertaken in Walton et al. (2012a, 2013a). We refer the reader to those works for a detailed description of this approach, but in brief, we include a narrow (intrinsic width of $\sigma=10 \mathrm{eV}$ ) Gaussian, and vary its energy across the 5-9 keV energy range in steps of $0.04 \mathrm{keV}$. The continuum model used is the DISKBB+SIMPL combination described above. For each line energy, we record the $\Delta \chi^{2}$ improvement resulting from the inclusion of the Gaussian line, as well as the best fit equivalent width (EW) and its 90 and $99 \%$ confidence limits, calculated with the EQWIDTH command in XSPEC, using 10,000 parameter simulations based on the best fit model parameters and their uncertainties.

The results obtained are shown in Figure 6; the top panel shows the $\Delta \chi^{2}$ improvement, and the limits on EW obtained are shown in the bottom panel. For clarity, we highlight the energies of the $K \alpha$ transitions of neutral, helium-like and hydrogen-like iron, as well as $\mathrm{EW}= \pm 30 \mathrm{eV}$, representative of the strongest iron absorption observed in GRS 1915+105 (Neilsen \& Lee 2009). As with our analysis of both Holmberg 


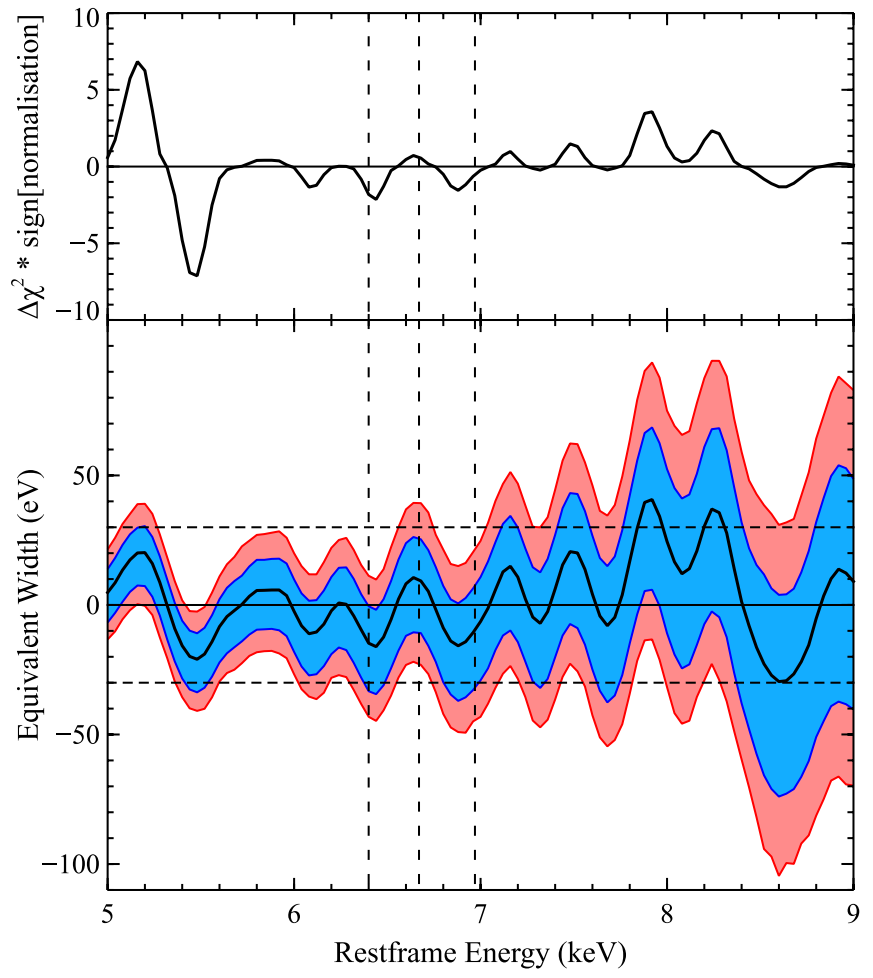

Figure 6. Top panel: the $\Delta \chi^{2}$ improvement obtained with the addition of a narrow Gaussian line, as a function of (rest frame) line energy, for the 2013 $X M M-N e w t o n+N u S T A R$ data set. Positive (negative) values of $\Delta \chi^{2}$ indicate the best fit line is in emission (absorption). We find no statistically significant narrow iron K features. Bottom panel: 90 (blue) and 99\% (red) confidence contours for the equivalent width of the narrow line, indicating the line strengths any undetected narrow features could yet have. For clarity, the rest frame transitions of neutral, helium-like, and hydrogen-like iron $(6.4,6.67$, and $6.97 \mathrm{keV})$ are shown with vertical dashed lines. We also plot dashed, horizontal lines representing $\mathrm{EW}= \pm 30 \mathrm{eV}$, roughly indicative of the absorption lines seen in the Galactic BHB GRS 1915+105 (Neilsen \& Lee 2009), for comparison.

(A color version of this figure is available in the online journal.)

IX X-1 and NGC 1313 X-1, we find no statistically significant line detections. Any narrow atomic features in the 2013 data in the immediate Fe $K$ band (6-7 keV) must have equivalent widths less than $\sim 50 \mathrm{eV}$ at $99 \%$ confidence. The line limits obtained here for Circinus ULX5 are not as stringent as those obtained most recently for Holmberg IX X-1 (Walton et al. 2013a), but are similar to those obtained previously for NGC 1313 X-1 (Walton et al. 2012a).

\subsection{Suzaku in 2006}

As with the more recent 2013 observations, the Suzaku spectrum obtained in 2006 is not well modelled by a simple absorbed power law $\left(\chi_{v}^{2}=1029 / 618\right)$, requiring an intrinsically curved continuum instead. This is apparent from Figure 7, in which the spectra from the three main epochs analyzed $(2001,2006,2013)$ are directly compared, after having been unfolded through the same simple model, consisting of just a constant. Indeed, the Suzaku data have a distinctly thermal-like appearance. However, while a DISKBB continuum gives a marked improvement and formally provides a statistically acceptable fit to the data $\left(\chi_{v}^{2}=639 / 618\right)$, an excess at high energies is again visible, broadly similar to XMM-Newton+NUSTAR data set considered previously, albeit apparently weaker and, by necessity, occurring at lower energies, owing to the limited bandpass of the XIS data.

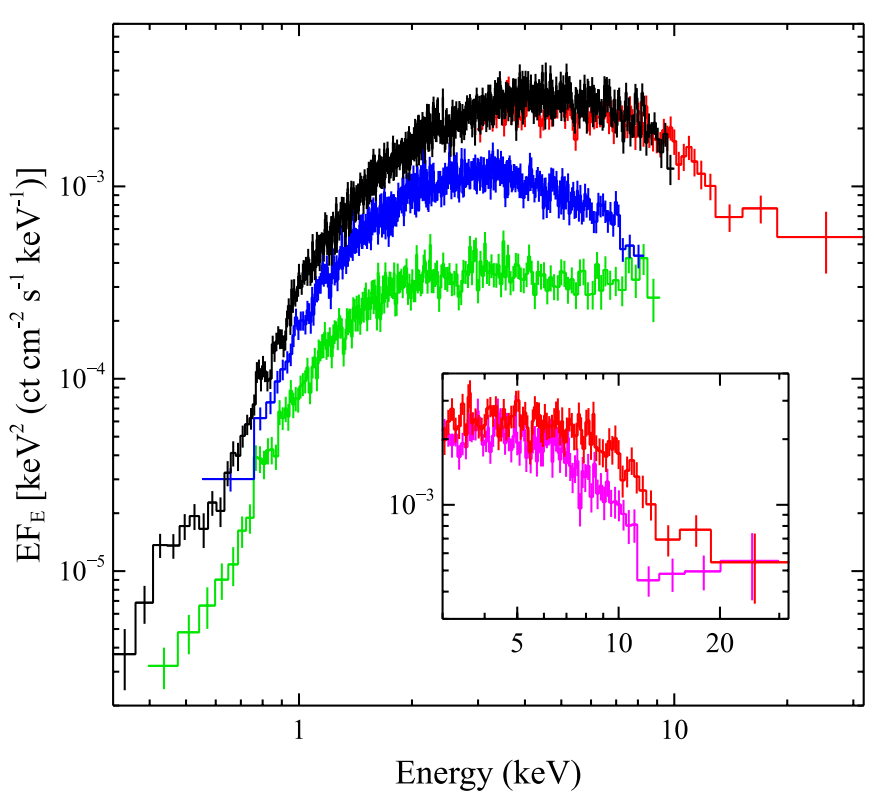

Figure 7. Spectral evolution displayed by Circinus ULX5. The 2013 $X M M$-Newton (EPIC-pn) and NuSTAR (FPMA) data are shown in black and red, respectively, while the 2006 Suzaku (FI XIS) and the 2001 XMM-Newton data are shown in blue and green. The additional NUSTAR data are also shown in the inset in magenta, compared to the same NUSTAR data set shown in the main figure. All the data have been unfolded through the same model, which simply consists of a constant.

(A color version of this figure is available in the online journal.)

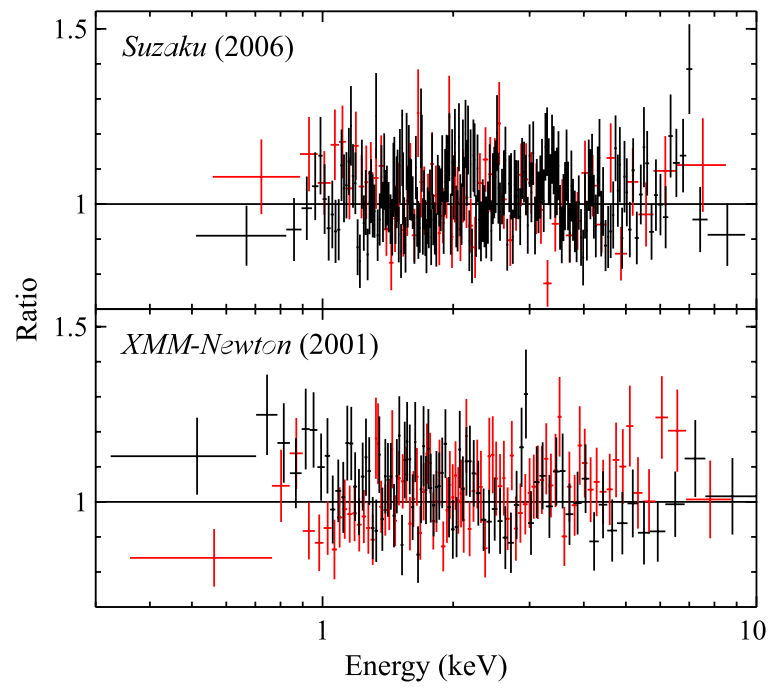

Figure 8. Data/model ratios for the DISKBB+SIMPL model applied to the 2006 Suzaku observation (top panel) and the 2001 XMM-Newton observation (bottom panel). Excellent fits are obtained in both cases (see Table 2). Front-illuminated XIS (EPIC-pn) and back-illuminated XIS (EPIC-MOS) data are shown in black and red respectively; the data have been rebinned for visual clarity.

(A color version of this figure is available in the online journal.)

In this case, the high energy excess can be resolved by allowing for a shallower radial temperature profile for the disc with DISKPBB ( $p=0.61 \pm 0.03)$, and an excellent fit is obtained $\left(\chi_{v}^{2}=606 / 617\right)$. However, this may be a consequence of the limited bandpass, so we also consider a Comptonisation origin for the high energy excess, again utilizing the DISKBB+SIMPL combination. Unsurprisingly, an excellent fit is also obtained with this model $\left(\chi_{v}^{2}=590 / 616\right.$; see Figure 8$)$, but due to the weak excess and the lack of high energy data, the SIMPL parameters are again highly degenerate and therefore only poorly 

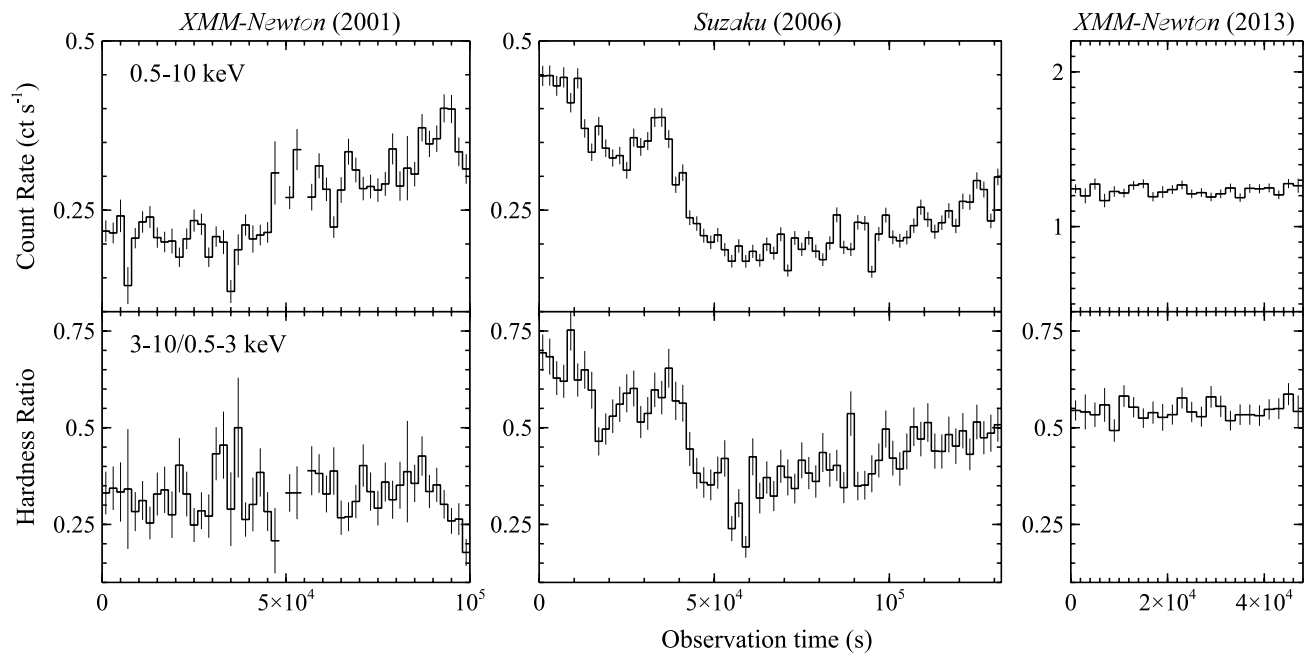

Figure 9. $0.5-10.0 \mathrm{keV}$ lightcurves (top panels) and 3-10/0.5-3.0 keV hardness ratios (bottom panels) for the 2001 XMM-Newton (left panels), 2006 Suzaku (center panels), and the 2013 XMM-Newton observations (right panels). The axes in the top panels have been scaled to show a similar dynamic range around the mean count rate for each observation. Contrasting short term behavior is clearly seen in each of these three observations (see text).

constrained individually. The observed $0.5-10.0 \mathrm{keV}$ flux during this epoch, $(2.90 \pm 0.04) \times 10^{-12} \mathrm{erg} \mathrm{cm}^{-2} \mathrm{~s}^{-1}$, is significantly lower than observed in early 2013 . Given the quality of fit obtained, the parameter degeneracy already present with this combination, and the lack of any obvious residuals, we do not consider the more complex DISKPBB+SIMPL, DISKBB+COMPTT, or COMPTT+COMPTT models here.

\subsection{XMM-Newton in 2001}

Again, for the early (2001) XMM-Newton observation, we begin by modelling the data with a simple absorbed power law model. Remarkably, in contrast to the two data sets considered so far, such a simple model actually provides an excellent fit to this data set $\left(\chi_{v}^{2}=587 / 580\right)$. Again, the results obtained for the spectral parameters are presented in Table 2. In contrast, the simple accretion disk model provides a very poor fit $\left(\chi_{v}^{2}=\right.$ $820 / 580$ ), with the model severely under predicting the data above $\sim 5 \mathrm{keV}$. This indicates there is a marked difference between this XMM-Newton observation and the later Suzaku and $X M M-N e w t o n+N u S T A R$ data sets, in which the spectrum below $10 \mathrm{keV}$ is generally well modelled with thermal emission. The difference can clearly be seen in Figure 7. While the Suzaku and XMM-Newton+NuSTAR data sets display curvature in the 3-10 keV bandpass, the 2001 XMM-Newton data set does not, indeed appearing more consistent with an absorbed, simple, power-law-like continuum. The observed $0.3-10.0 \mathrm{keV}$ flux in this observation is $(1.93 \pm 0.04) \times 10^{-12} \mathrm{erg} \mathrm{s}^{-1}$, slightly lower again than the Suzaku data set, and significantly lower than the $X M M-N e w t o n+N u S T A R$ data set.

For completeness, we also apply some of the other models that were previously considered. Statistically, the DISKPBB model offers a substantial improvement on the simpler DISKBB model, however the parameters are pushed to truly extreme values ( $T_{\text {in }} \simeq 5.0 \mathrm{keV}, p<0.5$ ) owing to the lack of curvature in the 3-10 keV bandpass. Given the flux of this observation, such an evolution of the disk would appear unphysical when compared to the more moderate parameters obtained with the other data sets. We also again consider the DISKBB+SIMPL combination in order to investigate the results obtained by interpreting the high energy ( $~(5 \mathrm{keV})$ excess observed with the DISKBB model alone as Comptonisation. As shown in Figure 8, this model gives an excellent fit $\left(\chi_{\nu}^{2}=569 / 578\right)$, and actually provides a reasonable improvement over the pure power law continuum $\left(\Delta \chi^{2}=18\right.$ for two additional free parameters). However, the model is again dominated by the power law tail provided by the SIMPL component, and we obtain a fully consistent photon index to the pure power law continuum. As with the Suzaku observation, given the quality of fit obtained with the DISKBB+SIMPL model, and the lack of any obvious residuals, we do not consider the more complex DISKPBB+SIMPL, DISKBB+COMPTT or COMPTT+COMPTT models for this data set, and conclude that it is best represented with a continuum dominated by a powerlaw-like component with $\Gamma \sim 2$, perhaps with some mild disc contribution at lower energies.

\section{SHORT TERM VARIABILITY}

Figure 9 shows the $0.5-10.0 \mathrm{keV}$ lightcurves for the three longest duration observations, as well as the evolution of the 3-10/0.5-3.0 keV hardness ratio during these observations. Contrasting short term behavior can be seen from each of the three observations. The long XMM-Newton observation in 2001 shows clear flux variability, with no strong associated spectral variability. The 2006 Suzaku observation also shows strong variability, although in this instance there is clear spectral variability. Indeed, this spectral variability appears to correlate extremely well with the source flux, as shown in Figure 10, with the source displaying harder spectra at higher fluxes (note that Circinus ULX5 is vastly below the pile-up limit for Suzaku). Finally, in 2013, Circinus ULX5 does not appear to show any strong short term flux or spectral variability at all, although we note that, of the three observations considered, this has the shortest duration. In order to quantify the differing levels of observed variability, we compute the fractional excess variance $\left(F_{\mathrm{var}}\right.$; Edelson et al. 2002; Vaughan et al. 2003) over the $0.5-10.0 \mathrm{keV}$ energy range for each of the long observations. For consistency, we divide the earlier XMM-Newton and Suzaku observations into two and three segments of $\sim 45-50$ ks duration respectively, roughly that of the latest $X M M$-Newton observation, and present the average value of $F_{\text {var }}$ obtained from these, in order to ensure we are comparing the same time scales for each data set. The values obtained are presented in Table 3, and confirm our earlier visual conclusions. 


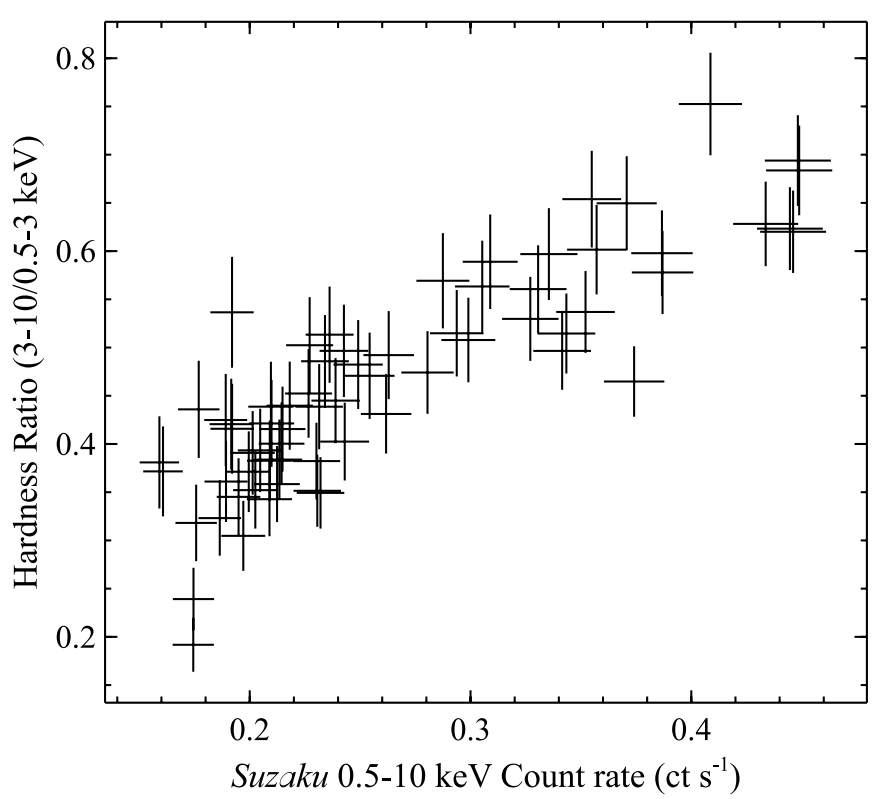

Figure 10. Hardness ratio-intensity diagram for the 2006 Suzaku observation. During this epoch, the 3-10/0.5-3.0 keV hardness ratio clearly correlates with the full $0.5-10.0 \mathrm{keV}$ count rate.

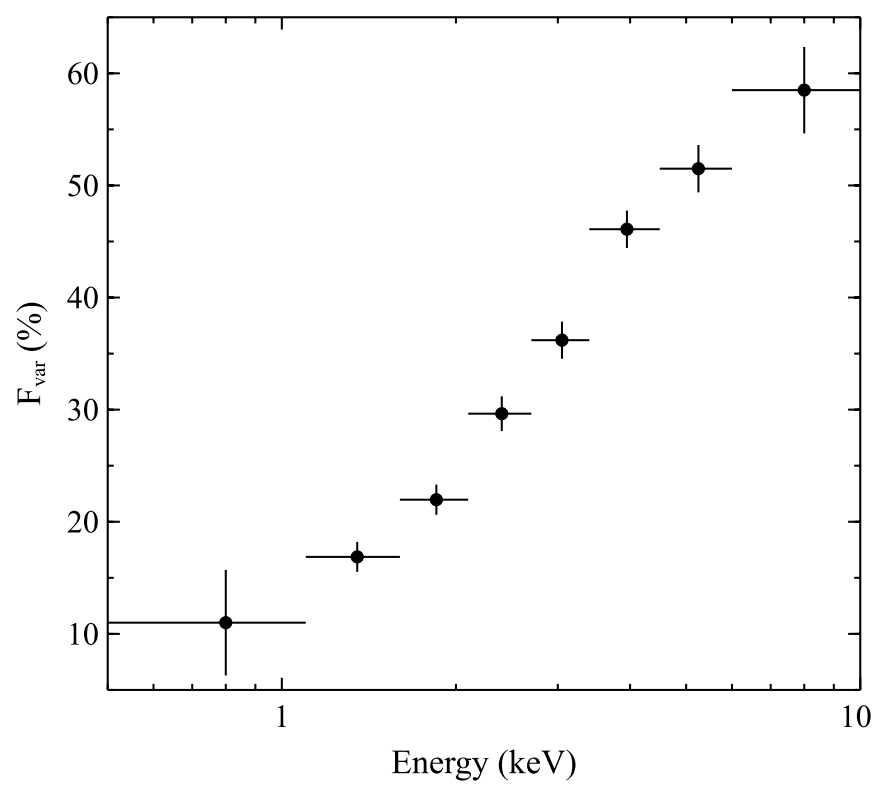

Figure 11. Fractional excess variance $\left(F_{\mathrm{var}}\right)$ as a function of energy for the 2006 Suzaku observation. $F_{\text {var }}$ clearly increases with increasing energy.

\subsection{Spectral Variability}

For the Suzaku observation, we also briefly investigate the nature of the observed spectral variability. First, we simply calculate the $F_{\text {var }}$ as a function of energy. The resulting variability spectrum is shown in Figure 11. It is clear that the fractional variability increases monotonically with increasing energy, and is strongest above the peak of the disk emission, at the energies at which the power-law-like tail is most prominent in the DISKBB+SIMPL model. Second, we split the observation into seven segments, $\sim 15-20 \mathrm{ks}$ in duration, and spectra are extracted for each, following the data reduction procedure outlined above (Section 2.2.2). These seven segments are modelled simultaneously with the DISKBB+SIMPL combination, in order to investigate the behavior of the thermal component. As

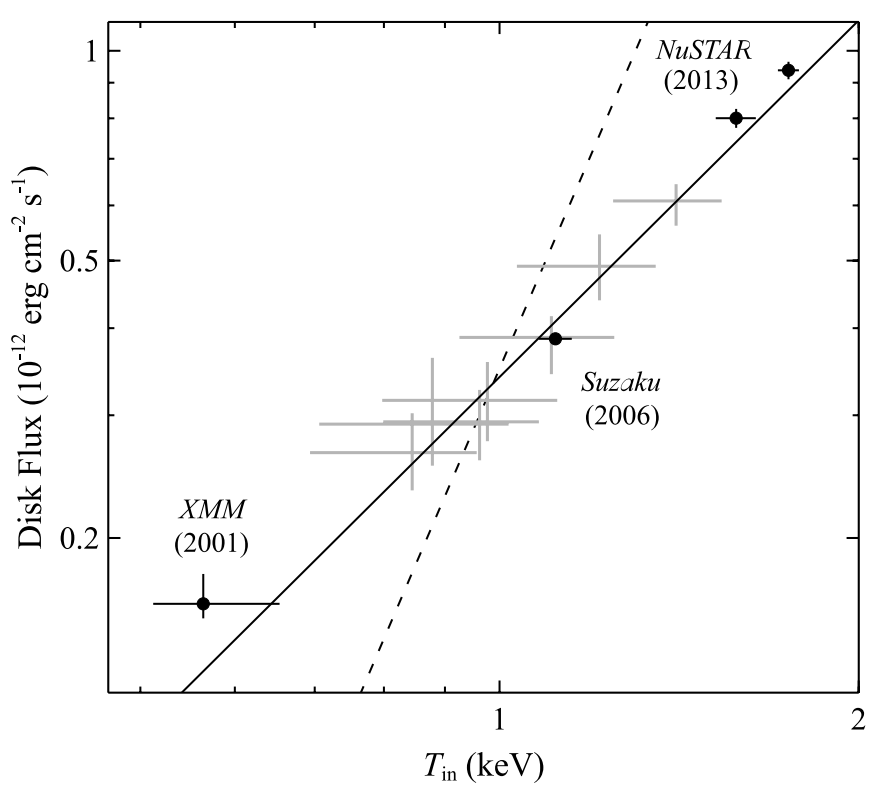

Figure 12. Luminosity-temperature relation inferred for the accretion disk of Circinus ULX5 when using the DISKBB+SIMPL model. The multi-epoch data are shown in black (see Section 5), while the individual Suzaku segments considered are shown in grey (see Section 4.1). Remarkably, despite probing very different time scales, all the data appear to follow a common relation, which is significantly shallower than the naively expected $L \propto T^{4}$ relation (shown as a dashed line) for standard stable disk emission. The solid line shows the best fit to the multi-epoch data.

Table 3

Observed 0.5-10.0 keV Fractional Excess Variability Amplitudes

\begin{tabular}{lcc}
\hline \hline Mission & ObsID & $\begin{array}{c}0.5-10.0 \mathrm{keV} \mathrm{F} \text { var } \\
(\%)\end{array}$ \\
\hline XMM-Newton & 0111240101 & $15 \pm 1$ \\
Suzaku & 701036010 & $12 \pm 1$ \\
XMM-Newton & 0701981001 & $<2$ \\
\hline
\end{tabular}

the SIMPL parameters were not well constrained when considering the full time averaged spectrum, we link the photon index between all the epochs in order to minimize the effects any parameter degeneracies might have on the disk parameters obtained, which we are primarily interested in. We also link the column densities of the intrinsic absorption between the segments, as there is no strong evidence that this varies here (see also Miller et al. 2013).

With this procedure, we obtain an excellent global fit to the seven segments, with $\chi_{v}^{2}=695 / 719$. In addition to the disk temperature, which is a direct product of the model, we also compute the intrinsic disk flux for each segment with CFLUX in XSPEC. As shown in Figure 12, there is a clear, positive correlation between the inferred flux and the temperature of the disk component. This evolution may also contribute to the energy dependence of the fractional variability. However, when modelled with a power law relation, i.e., $L \propto T_{\text {in }}^{\alpha}$, accounting for the uncertainties on both the temperatures and the fluxes with the algorithm described in Williams et al. (2010), the exponent obtained is much shallower than the expected $L \propto T^{4}$ relation for a standard thin accretion disc with a constant emitting area, and a constant color correction factor: $\alpha=1.74 \pm 0.34(1 \sigma$ uncertainty). 


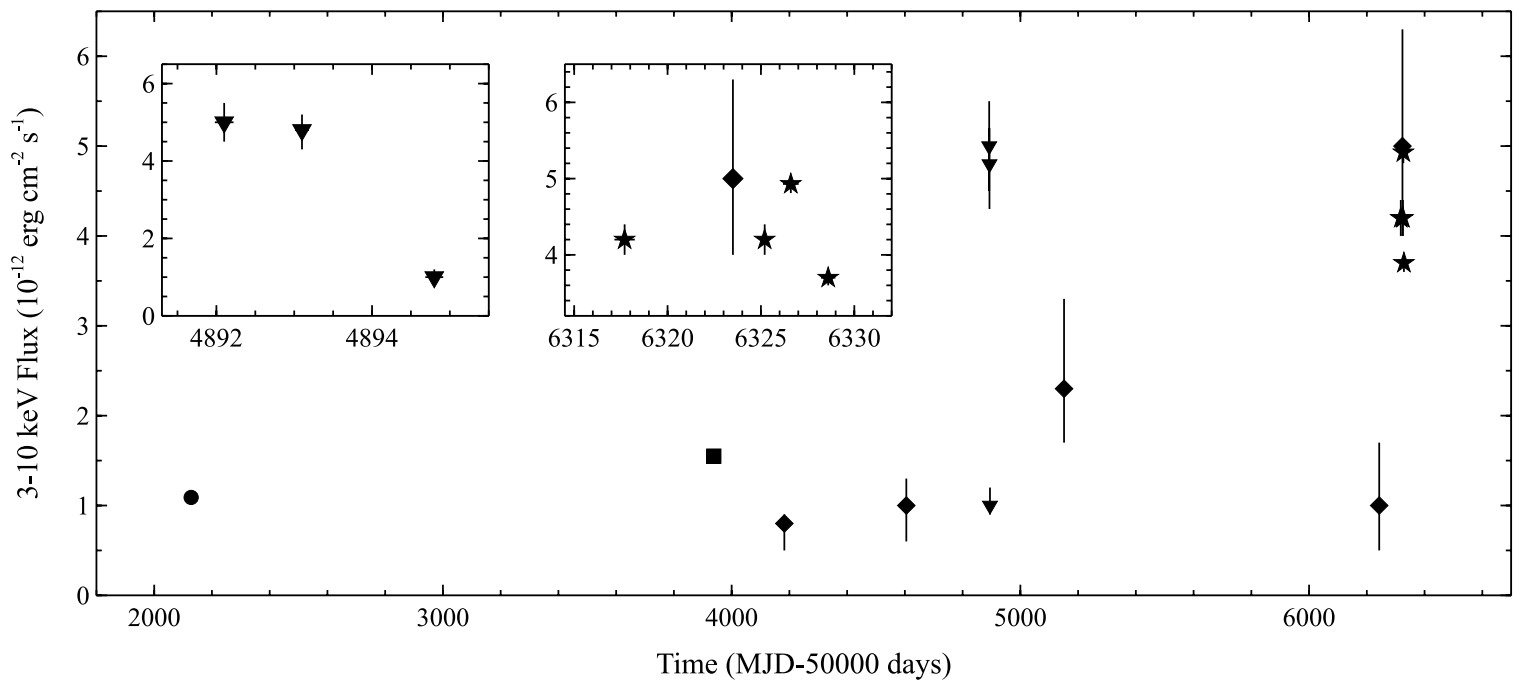

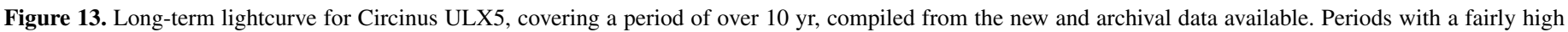

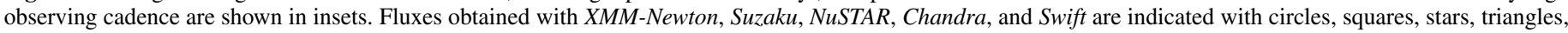
and diamonds respectively.

\section{LONG TERM EVOLUTION}

Given the observed correlation between the luminosity and temperature of the disk component based on the short term variability during the 2006 Suzaku observation, we now wish to test whether this correlation also holds for the long-term spectral evolution observed. Therefore, we take the same approach outlined previously (Section 4.1) in order to investigate the evolution of the disk parameters, but here making use of multi-epoch data. In addition to the higher $\mathrm{S} / \mathrm{N}$ data sets previously analyzed, we also now consider the other two pointed NUSTAR observations, obtained on either side of the observation coordinated with XMM-Newton (ObsIDs 30002038002 and 30002038006). The spectra from these two observations show some spectral and flux evolution when compared to the NuSTAR data previously presented (see inset in Figure 7), but are broadly consistent with one-another, and so we combine them into a single data set for the purposes of this analysis.

This approach also provides an excellent global fit to the average spectrum from each epoch considered, with $\chi_{v}^{2}=2486$ / 2484 . The common column density obtained from all the data sets is $N_{\mathrm{H} \text {;int }}=1.9_{-0.1}^{+0.2} \times 10^{21} \mathrm{~cm}^{-2}$, and the common photon index obtained is $\Gamma=2.3_{-0.2}^{+0.1}$. Remarkably, as is clear from Figure 12, we find that despite the observations being taken over a span of more than a decade, the multi-epoch evolution of the disk component is fully consistent with an extrapolation of the short-term evolution observed from the Suzaku data alone. Modelling the data again with a power law relation, the multiepoch exponent obtained is $\alpha=1.70 \pm 0.17$, fully consistent with that obtained previously.

\subsection{Flux Evolution}

In addition to our analysis of the higher quality data sets available for Circinus ULX5, we also present a brief analysis of the lower S/N observations (the Swift snapshots, the short Chandra observations, and the initial NUSTAR detection) in order to build up a long term lightcurve. Given the spectral evolution apparent in Figure 7, we model each of these data sets with a phenomenological power law continuum with a variable high-energy exponential cutoff, in order to allow for either curved or power-law-like continua, as favored by each individual data set. The neutral absorption is treated in the same manner as our more detailed spectral analysis, including both a Galactic and an intrinsic absorption component. Given the moderate quality (and/or the high energy nature) of the data, the intrinsic column is fixed at $N_{\mathrm{H} \text {;int }}=2.5 \times 10^{21}$ atom cm $\mathrm{cm}^{-2}$, broadly consistent with the results obtained from the higherquality data sets. In the case of the Swift observations, spectral fitting is performed through minimization of the Cash statistic (Cash 1979) owing to the much less stringent rebinning applied to these data (see Section 2.2.4).

With this simple model, we compute the observed flux for these additional exposures in the energy band, common to all the missions utilized in this work, 3-10 keV. The fluxes obtained are quoted in Table 1, and the long term lightcurve is shown in Figure 13. Although the lightcurve is sparsely sampled given the overall span of over a decade, strong long-term variability is clearly apparent, with the $3-10 \mathrm{keV}$ flux varying by at least a factor of $\sim 5$, and there are two clear periods of high flux observed in early 2009 (Chandra data sets) and early 2013 (NuSTAR data sets). A more comprehensive monitoring campaign on this source would be highly beneficial, and would allow us to assess how frequently such high flux states occur.

\section{DISCUSSION}

\subsection{Association with Circinus}

Throughout this work, we have assumed that Circinus ULX5 is associated with the Circinus galaxy. Here, we present a brief discussion of whether this is likely to be the case, or whether Circinus ULX5 could plausibly be explained as distant background active galaxy, or, particularly given the low Galactic latitude of Circinus, as a foreground Galactic source.

First, we stress that the broadband X-ray spectrum of Circinus ULX5 obtained in 2013 is not consistent with that of background AGNs, which typically display power law spectra (e.g., Piconcelli et al. 2003). Unfortunately, at the time of writing there is no Hubble coverage at the position of Circinus ULX5 with which to perform a detailed search for optical counterparts which could assist in classifying this source (e.g., Heida et al. 2013). Instead, we have searched for possible mid-infrared (MIR) counterparts in the wide-field Spitzer (Werner et al. 2004) Infrared Array 

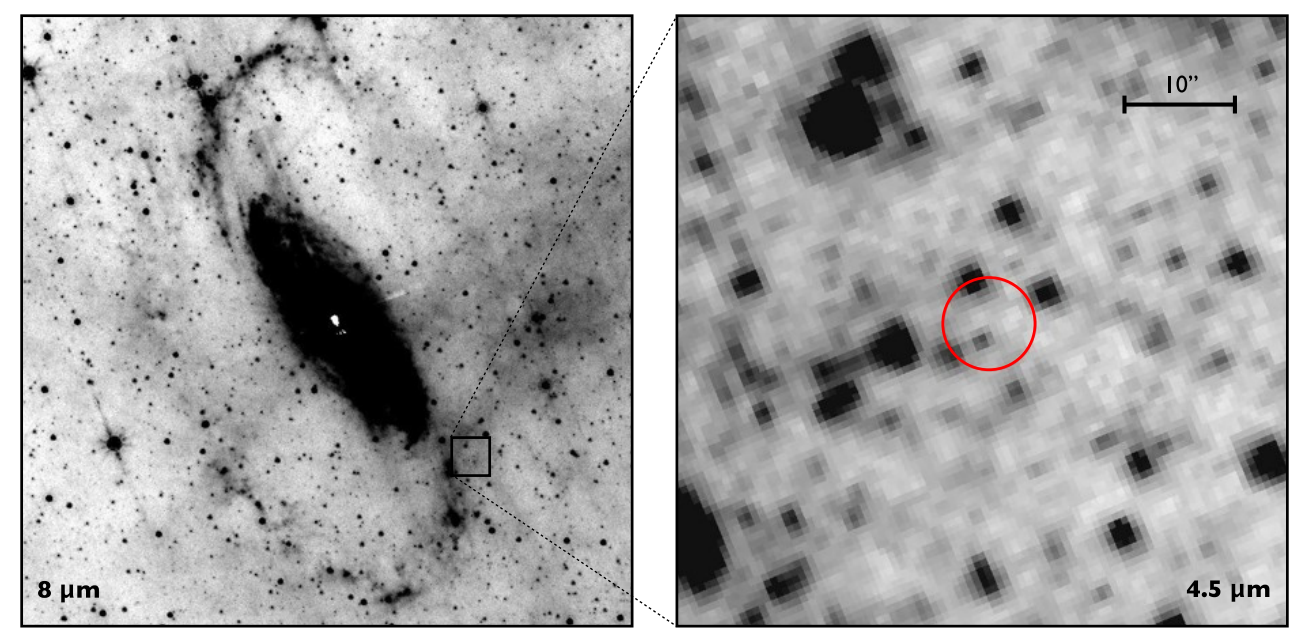

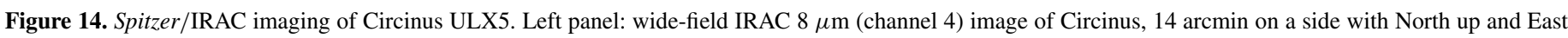

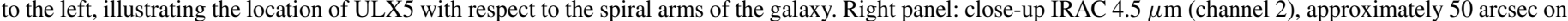
a side, centered on ULX5. The red circle, with radius 4 arcsec, is centered on the $N u S T A R$ position of ULX5 $\left(\right.$ R.A. $=14^{\mathrm{h}} 12^{\mathrm{m}} 39^{\mathrm{s}}$, decl. $\left.=-65^{\circ} 23^{\prime} 34^{\prime \prime}\right)$.

(A color version of this figure is available in the online journal.)

Camera (IRAC; Fazio et al. 2004) map of the Circinus galaxy obtained by For et al. (2012). Figure 14 shows MIR images of Circinus, with a wide-area $8 \mu \mathrm{m}$ view to highlight that Circinus ULX5 resides near one of the spiral arms of this galaxy, and a $4.5 \mu \mathrm{m}$ zoom-in of the X-ray position with a $4^{\prime \prime}$ radius circle, illustrative of a conservative estimate for the position uncertainty. There is one MIR source within this circle and an additional three MIR sources in close vicinity. All four sources are well detected in channel $1(3.6 \mu \mathrm{m})$ and channel $2(4.5 \mu \mathrm{m})$ of IRAC, and have relatively blue colors across this bandpass. In the Vega system, [3.6]-[4.5] $\approx 0$ for all four sources, consistent with Galactic stars and inconsistent with background AGNs which typically have red MIR colors (e.g., Stern et al. 2005). Therefore, we do not consider it likely that Circinus ULX5 is a distant AGN being misidentified as a ULX. Greater positional accuracy through a dedicated on-axis Chandra observation will be required to determine which, if any, of the Spitzer sources is the true NIR counterpart to Circinus ULX5, and to aid in future searches for optical counterparts.

The obvious candidates for Galactic sources that could also masquerade as a bright ULX are foreground X-ray binaries (XRBs). As the Circinus galaxy is roughly in the direction of the Galactic center, the extent of the Galactic plane toward Circinus ULX5 is $\sim 20 \mathrm{kpc}$ given our own location within the Galaxy (Sale et al. 2010). Jonker \& Nelemans (2004) find that the typical scale-height of XRBs out of the Galactic plane is roughly less than $1 \mathrm{kpc}$, which contributes a negligible amount to the maximum distance Circinus ULX5 could be at if within our Galaxy. Even at the highest observed flux in 2013, Circinus ULX5 would therefore have a luminosity of $L_{\mathrm{X}} \lesssim 3 \times 10^{35} \mathrm{erg} \mathrm{s}^{-1}$ if Galactic, equivalent to $L_{\mathrm{X}} / L_{\mathrm{E}} \lesssim 10^{-4}$ for a $10 M_{\odot}$ black hole. Furthermore, these observations revealed the source to have a very soft broadband spectrum. Although Galactic BHBs are known to display soft spectra, these are observed at high luminosities $\left(L_{\mathrm{X}} / L_{\mathrm{E}} \gtrsim 0.1\right)$. In contrast, low luminosity Galactic BHBs are generally observed to have hard spectra (e.g., Remillard \& McClintock 2006). Galactic neutron star XRBs can also display soft broadband spectra, similar to that observed, but as with Galactic BHBs, these are observed at high luminosities ( $L_{\mathrm{X}} \gtrsim 5 \times 10^{36} \mathrm{erg} \mathrm{s}^{-1}$; Barret 2001).

Finally, a Galactic cataclysmic variable (CV) would be consistent with the low luminosity required to place the source in our own Galaxy. However, the observed spectrum does not appear to be consistent with those of known CVs. The high energy X-ray emission from CVs is generally observed to arise from a multi-temperature collisionally ionized plasma, either from the boundary layer between the accretion disc and the white dwarf surface for non-magnetic CVs, or from the postshock plasma in the accretion columns for magnetic CVs; see Mukai (2005) and Kuulkers et al. (2006) for a recent reviews. However, as shown in Section 3.1.3, the iron emission expected from such a plasma, which is reliably observed in known CVs (see Middleton et al. 2012 for a particularly extreme case), is not observed from Circinus ULX5. Indeed, fits to the high energy spectrum with thermal plasma models (e.g., Raymond \& Smith 1977) with solar abundances fail completely. Furthermore, the spectral evolution shown in Figure 7 is not typical behavior for a Galactic CV.

Therefore, we conclude that none of these classes of Galactic $\mathrm{X}$-ray source offers an obvious observationally self-consistent scenario for Circinus ULX5. A further point worth highlighting, throughout all our spectral modelling we always require an absorption column in excess of the Galactic column (as given in Kalberla et al. 2005), both when considering the local average for the column, and the measurement closest to the source position, which also argues against a Galactic origin for Circinus ULX5. In combination with the arguments against a distant AGN origin presented above, this strongly supports its association with Circinus. Finally, we note that the observed variability rules out a young supernova remnant scenario for Circinus ULX5, leaving an extreme ULX (peak luminosity of $L_{\mathrm{X}} \sim 2 \times 10^{40} \mathrm{erg} \mathrm{s}^{-1}$ ) as the only plausible interpretation. In addition, as discussed in the following sections, Circinus ULX5 does display numerous similarities with other ULXs that radiate at $L_{\mathrm{X}} \geqslant 10^{40} \mathrm{erg} \mathrm{s}^{-1}$, further supporting this conclusion.

\subsection{Extreme Ultraluminous X-Ray Sources}

Extreme ULXs with $L_{\mathrm{X}} \geqslant 10^{40} \mathrm{erg} \mathrm{s}^{-1}$ are rare, with only a few dozen identified (Walton et al. 2011b; Swartz et al. 2004, 2011). Furthermore, many of these sources are reasonably distant $(D>10 \mathrm{Mpc}$; Walton et al. 2011b); extreme ULXs close enough to enable detailed study are rarer still. In this work, we have presented a multi-epoch spectral and temporal analysis of one of the few such ULXs known, Circinus ULX5, 
which, to date, has received very little observational attention, appearing only in the catalogue of Winter et al. (2006). This is, in part, likely due to its location with respect to the Circinus galaxy, sitting outside the D25 isophote in the relative outskirts of the galaxy, where the chances of an observed source being foreground/background are fairly high. However, as previously outlined, the association of this source with the Circinus galaxy appears to be robust.

Observationally, Circinus ULX5 appears to show a number of similarities with other extreme ULXs. First of all, the high quality data sets available when the source was fairly bright show clear curvature in the 3-10 keV bandpass (see Figure 2). Such curvature is frequently observed in the spectra of other bright ULXs (e.g., Stobbart et al. 2006; Gladstone et al. 2009; Walton et al. 2011a, 2013a; Bachetti et al. 2013), and does not seem to be consistent with the power-law-like emission expected from a standard, optically thin sub-Eddington corona.

In addition, based on the compilation of serendipitous detections available, it is clear that Circinus ULX5 can vary in flux from epoch to epoch by at least a factor of $\sim 5$ (see Figure 13 and Table 1). This, too, is broadly similar to the level of long-term variability displayed by other ULXs. For example, the recent Swift monitoring campaigns on Holmberg IX X-1 and NGC 5907 ULX1 revealed long term variability by a factor of 3-4 (Kong et al. 2010; Sutton et al. 2013), and multi-epoch XMM-Newton observations of NGC 1313 X-1 reveal long term variability by a similar factor of $\sim 3$ (Feng \& Kaaret 2006). One potentially subtle difference is that many of the brighter ULXs appear to show suppressed, short-term variability within single observations (i.e., time scales less than $\sim 100 \mathrm{ks}$; Heil et al. 2009). In this case, we were fortunate enough to observe such variability from Circinus ULX5, particularly during the 2006 Suzaku observation. However, it is noteworthy that during the most recent observation of reasonable duration, when Circinus ULX5 was at its brightest, the short-term variability also appeared to be suppressed, similar to the results presented by Heil et al. (2009). Furthermore, high quality observations of ULXs with which variability can properly be studied are relatively rare, so in many cases the multi-epoch evolution of the short-term variability is not well constrained, and may well be similar to that observed here.

\subsection{The Broadband X-Ray Spectrum of Circinus ULX5}

The NUSTAR observations of Circinus ULX5, along with the observations of NGC 1313 X-1, IC 342 X-1 and Holmberg IX X-1 (see M. Bachetti et al. 2013; V. Rana et al. and D. J. Walton et al., in preparation, respectively), represent one of the first times it has been possible to reliably constrain the spectrum of an extreme ULX above $10 \mathrm{keV}$. In combination with $X M M-N e w t o n$, we have been able to constrain the broadband spectral form of Circinus ULX5 over the $0.3-30.0 \mathrm{keV}$ bandpass during a historically high flux state. During this epoch, Circinus ULX5 displayed numerous observational similarities at lower energies $(\leqslant 10 \mathrm{keV})$ to previous observations of other extreme ULXs, as outlined above.

The key spectral similarity is the curvature observed in the continuum over the 3-10 keV energy range. Based on the available data, which was, until recently, limited to below $10 \mathrm{keV}$, a variety of interpretations for this curvature have been proposed in the literature, including a high temperature accretion disk (Watarai et al. 2001), optically thick Comptonisation in a cool corona (Gladstone et al. 2009), and relativistic disk reflection (Caballero-García \& Fabian 2010). The former two predict that the curvature should continue to higher energies, falling off with a thermal Wien tail, while the latter predicts a much stronger high energy spectrum owing to the Compton reflection hump (see Walton et al. 2011a).

Broadly similar to the other ULXs observed by NuSTAR to date, the emission above $10 \mathrm{keV}$ from Circinus ULX5 is fairly weak compared to the emission below $10 \mathrm{keV}$ during this epoch. The spectrum peaks at $\sim 4-5 \mathrm{keV}$, and then falls away fairly steeply. However, when the broadband XMM-Newton $+N u S T A R$ spectrum of Circinus ULX5 is fitted with any purely thermal model with a Wien spectrum at high energies, the NuSTAR data shows a clear excess over the model at high energies (see Figure 3); pure thermal models can therefore be rejected in this case. Nevertheless, the excess is not strong enough to be explained as the Compton hump if the $3-10 \mathrm{keV}$ curvature is due to relativistic iron features from the inner accretion disk, so disk reflection does not appear to offer a viable solution in this case either. Instead, this high energy excess is well modelled simply as a power law tail to the lower energy curved spectrum. In physical terms, the broadband spectrum can be well explained as a relatively hot accretion disk with an additional high energy Comptonised tail, or as a Comptonised spectrum from an optically thick corona of electrons with a dual temperature distribution. However, given the more straightforward comparison with the established disk-corona Galactic BHB paradigm, and the multi-epoch spectral and variability properties observed, we prefer the former.

\subsection{An Emerging/Re-emerging Accretion Disk Scenario}

One of the most striking aspects of the available data for Circinus ULX5 is the clear spectral variability (see Figure 7). During the 2001 XMM-Newton observation, the source appears to display a power law spectrum, with no apparent curvature across the 3-10 keV energy range, while in the higher luminosity observations obtained with Suzaku in 2006 and XMMNewton $+N u S T A R$ in 2013, the spectrum seems to be dominated by a thermal component, displaying the curvature across the 3-10 keV bandpass now typically associated with bright ULXs. This rather dramatic spectral evolution is reminiscent of the sub-Eddington state transitions observed in Galactic BHBs (e.g., Remillard \& McClintock 2006; Done et al. 2007; Fender \& Belloni 2012 for reviews). In particular, the evolution from the 2001 $X M M-N e w t o n$ data to the later thermal-like observations (2006, 2013) seems to be comparable to the transition from the hard/ hard-intermediate state, in which the spectrum is largely dominated by the Comptonised emission from the corona, to the soft state, in which the spectrum is dominated by the thermal emission from the accretion disk.

Observation of spectral state transitions in ULXs have been claimed for a number of individual sources in the literature (e.g., Feng \& Kaaret 2006). However, in many of these cases, the claims relate to relatively subtle changes in the observed spectrum, rather than true evidence for state transitions in the traditional sense displayed by Galactic BHBs, particularly when one considers that the same state can be observed in the same source over a range of luminosities. Nonetheless, there are some ULXs that show spectral evolution as strong as observed in Circinus ULX5. The most compelling case for subEddington transitions is also the most luminous ULX currently known, ESO 243-49 HLX-1 (Farrell et al. 2009), with strong spectral evolution that follows the characteristic state-transition cycle displayed by Galactic BHBs in outburst (Servillat et al. 2011). However, in many respects ESO 243-49 HLX-1 is rather 


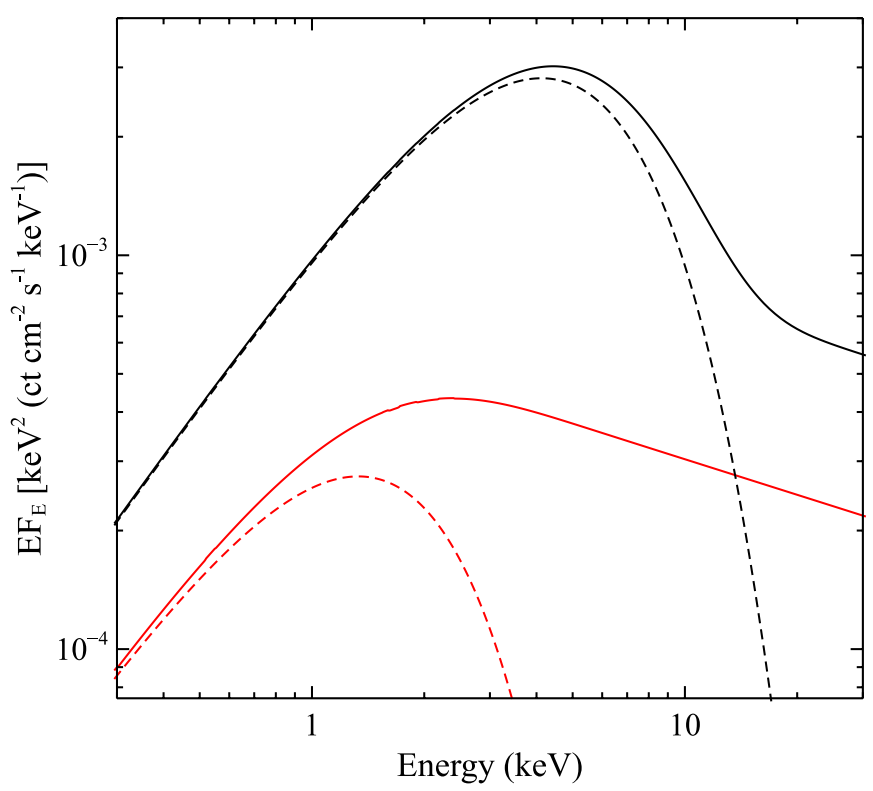

Figure 15. Total (unabsorbed) model (solid lines) and the relative contribution of the accretion disk (dashed lines) for the 2013 XMM-Newton+NuSTAR (black) and 2001 XMM-Newton (red) observations, obtained with our joint analysis of all the high $\mathrm{S} / \mathrm{N}$ data sets with the DISKBB+SIMPL model (see Section 5). The relative contribution of the accretion disk over the analyzed $0.3-30 \mathrm{keV}$ energy range is much greater in the high flux case.

(A color version of this figure is available in the online journal.)

unique, displaying apparently periodic outburst cycles over which the flux varies by more than an order of magnitude (Lasota et al. 2011; Godet et al. 2012), and the peak luminosity $\left(L_{\mathrm{X}} \sim 10^{42} \mathrm{erg} \mathrm{s}^{-1}\right)$ is vastly in excess of that reached by the majority of ULXs, so we caution against drawing strong comparisons between ESO 243-49 HLX-1 and the rest of the ULX population, including Circinus ULX5. However, amongst the more "standard" ULX population there have been a few cases in which the spectral evolution appears similar to that presented here, notably for the ULXs in IC 342 (Kubota et al. 2001b, 2002).

Indeed, although we have also considered more complex models, the high quality spectra available from multiple epochs are all well modelled with a simple combination of an accretion disc and a high energy Comptonised tail. As the source becomes more luminous, the accretion disc becomes more prominent with this combination, broadly similar to the behavior observed from Galactic BHBs. This is emphasized in Figure 15, which shows both the total model and the relative DISKBB contribution for the 2013 XMM-Newton+NuSTAR and the 2001 XMM-Newton data sets (i.e., the limiting flux cases) obtained with our joint analysis of all the high $\mathrm{S} / \mathrm{N}$ data sets with the DISKBB+SIMPL model combination (see Section 5). The variability behavior observed from Circinus ULX5 would also appear to support this evolution, when considered in comparison to Galactic BHBs. Clear short-term variability is observed in the two lower flux observations, in which the coronal emission is most prominent. In particular, during the Suzaku observation, we see that the fractional variability is stronger at higher energies (see Figure 11), where the coronal emission dominates. In contrast, during the highest flux observation, in which the disk emission dominates below $10 \mathrm{keV}$, no short-term variability is observed. In Galactic BHBs, short-term variability is also associated with strong coronal emission (e.g., Homan et al.
2001; Churazov et al. 2001), with the harder states generally displaying strong variability. In contrast, the thermal-dominated states display very little variability, with the emission dominated by a relatively stable accretion disk. The behavior of Circinus ULX5 is strikingly similar.

However, identifying the spectral evolution in Circinus ULX5 with the hard-to-soft state transition as seen in Galactic BHBs is not necessarily straightforward. In Galactic sources, hard states (the canonical low/hard state and the hard-intermediate state) can be observed at luminosities up to roughly $10-30 \%$ of the Eddington limit $\left(L_{\mathrm{E}}\right)$. If we identify the luminosity observed in the $2001 X M M-N e w t o n$ observation with this Eddington ratio (i.e., $L_{\mathrm{X}} / L_{\mathrm{E}}=0.3$, to be relatively conservative), the implied black hole mass is $M_{\mathrm{BH}} \gtrsim 90 M_{\odot}$. For the same spin, Eddington ratio and color correction factor, the accretion disc temperature of such a black hole should be a factor of at least $\sim 1.7$ cooler than for a black hole of mass $10 M_{\odot}$. Accretion disks observed from Galactic BHBs in the classical hard state are already quite cool, with $T_{\text {in }} \sim 0.2 \mathrm{keV}$ (e.g., Reis et al. 2009, 2010; Reynolds $\&$ Miller 2013). However, the disk temperatures obtained here are actually rather similar to those observed from such subEddington Galactic BHBs at higher luminosities: $T_{\text {in }} \sim 0.5 \mathrm{keV}$ in the intermediate states and $\sim 1-2 \mathrm{keV}$ in the disk-dominated states (see Reynolds \& Miller 2013).

Furthermore, although there is a clear positive correlation between the temperature and inferred luminosity of the disk (see Figure 12), which remarkably seems to hold across all the time scales and luminosities currently probed, the observed relation is $L \propto T_{\text {in }}^{\alpha}$ with $\alpha=1.78 \pm 0.19$, much shallower than the theoretically expected relation for a standard, geometrically stable thin disk (i.e., $L \propto T^{4}$ ). While Galactic BHBs do frequently show significant deviations from this relation (e.g., Dunn et al. 2011; Reynolds \& Miller 2013), the strongest deviations tend to be seen either when the coronal emission was strong (i.e., hard states), probably linked to strong irradiation of the disk, or very high luminosities, at which the scale height of the disk should start to increase and advection becomes increasingly important. During the thermal-dominated state, and while the disk remains thin, the observed luminositytemperature relation does tend to follow expectation relatively well (see also Gierliński \& Done 2004). At low luminosities, the deviations away from $L \propto T^{4}$ tend to be in the sense that the disk temperature is less dependent on the luminosity, becoming almost constant (Reynolds \& Miller 2013). In contrast, the Circinus ULX5 disk temperature displays a stronger dependence on luminosity than expected.

This is instead more similar to the behavior observed from another ULX, NGC 1313 X-2, based on the results obtained by Kajava \& Poutanen (2009) modelling XMM-Newton and Chandra data with a pure DISKBB model $(\alpha=2.39 \pm 0.16)$. It is also similar to the deviations from $L \propto T^{4}$ displayed by the Galactic BHBs GRO J1655-40 and XTE J1550-564 during the higher luminosity stages of their respective outbursts, when the sources were in the very high state (Kubota et al. 2001a; Kubota \& Makishima 2004; Saito et al. 2006). Sources in the very-high state also display a strong Comptonised continuum, but with steeper spectra and at substantially higher luminosities than the more traditional hard states (Remillard \& McClintock 2006). However, it is interesting to note that, for the latter cases, these deviations are still seen at sub-Eddington luminosities. XTE J1650-500, another sub-Eddington Galactic BHB, also seems to display similar behavior, although these data are more limited (Gierliński \& Done 2004). 
The observed accretion disk evolution can depart from the naively expected $L \propto T^{4}$ relation for a variety of reasons, which can be roughly separated into changes in the disk geometry (i.e., emitting area) and changes in the detailed plasma physics of the disk atmosphere (e.g., temperature dependent opacities, evolving vertical structure, dissipation profiles, etc.). The former primarily relates to changes in the inner radius, $R_{\text {in }}$, while the latter potentially incorporates a variety of complex effects that are difficult to isolate observationally. Therefore, the combined effect is instead typically quantified as a multiplicative color-correction factor that relates the effective mid-plane temperature of the disk to that actually observed: $T_{\text {in }}=f_{\text {col }} T_{\text {eff }}$. For simplicity, $f_{\text {col }}$ is usually assumed to be energy independent, such that is only serves to shift the observed temperature of the disc, rather than modify its spectral form. A substantial body of work has been undertaken attempting to theoretically determine the expected values of $f_{\text {col }}$ across a wide range of accretion regimes (e.g., Shimura \& Takahara (1995); Merloni et al. (2000); Fabian et al. (2004); Davis et al. (2005)), which typically suggest that $f_{\text {col }} \sim 1.7$ for disk-dominated sub-Eddington accretion. Here we are primarily interested in the relative, rather than the absolute behavior of $f_{\text {col }}$. The disk should evolve as $L \propto R_{\text {in }}^{2} T_{\text {eff }}^{4}$, hence variation in either $R_{\text {in }}$ or $f_{\text {col }}$ can result in the relation between the luminosity and the observed temperature deviating from $L \propto T_{\text {in }}^{4}$. In order to recover the luminosity-temperature relation observed for Circinus ULX5, either the inner radius of the disk must decrease with increasing luminosity as $R_{\text {in }} \propto L^{-0.6}$ (for a constant $f_{\text {col }}$ ), or the color-correction factor must increase with luminosity as $f_{\text {col }} \propto L^{0.3}$ (for a constant $R_{\text {in }}$ ), or some combination of these effects is present (observationally, changes in $R_{\text {in }}$ and $f_{\text {col }}$ are unfortunately highly degenerate, particularly for the simple models employed here). Therefore, given the inferred range in disk luminosity, either $R_{\text {in }}$ decreased by up to a factor of $\sim 2.9$, or $f_{\text {col }}$ increased by up to a factor of $\sim 1.7$ between the 2001 and 2013 observations.

If we invoke a variable $R_{\text {in }}$ to explain the observed spectral evolution, the implication is that the disk was truncated beyond the innermost stable circular orbit, at least during the lower flux observations. Substantial truncation of the accretion disk is only really expected at very low accretion rates $\left(L / L_{\mathrm{E}} \lesssim 10^{-2}\right)$, in the low-luminosity regime of the hard state (e.g., Tomsick et al. 2009). However, while the spectrum of Circinus ULX5 during the 2001 XMM-Newton observation could be considered relatively hard, particularly in comparison to the more recent observations, and can be modeled simply as a power law, it does not seem consistent with expectations for such a low-Eddington regime, during which Galactic BHBs typically display very hard spectra $(\Gamma \lesssim 1.7)$. Instead, the photon index obtained would suggest one of the higher luminosity hard state manifestations (if the source was in this regime at all). While it could be debated whether a factor of $\sim 3$ change in inner radius is "substantial" truncation, studies into the evolution of the relativistic iron line profiles observed from Galactic BHBs at various stages of their outbursts seem to rule out changes in the inner radius, even of this magnitude, as sources evolve from the higher luminosity hard states to the soft state (Reis et al. 2011; Walton et al. 2012b). A variable $f_{\text {col }}$ has been proposed as an alternative explanation for the continuum evolution seen at these luminosities (e.g., Reynolds \& Miller 2013; Salvesen et al. 2013). In any case, as stated previously, the observed behavior seems to compare far more favorably to high-luminosity observations of Galactic $\mathrm{BHBs}$, where disk truncation is not expected, but evolution in the vertical structure of the disk is. Therefore, it seems likely that the deviation away from $L \propto T_{\text {in }}^{4}$ is driven by a variable $f_{\text {col }}$ in this case.

The identification of Circinus ULX5 as a (reasonably) highEddington BHB system actually appears to provide a fairly selfconsistent picture, at least to first order, when considered in the context of the observed/expected behavior of Galactic BHBs. As previously discussed, the observed luminosity-temperature relation appears extremely similar to that inferred from known Galactic binaries at high luminosity, and seems to require a color-correction factor that increases with increasing luminosity. Theoretical considerations of the expected evolution of $f_{\text {col }}$ suggest that the opposite trend should be seen at low luminosities (Merloni et al. 2000), which appears to be supported by observation (Reynolds \& Miller 2013; Salvesen et al. 2013). Furthermore, the spectrum during the 2001 XMM-Newton observation, which shows strong Comptonised emission, is remarkably similar to that observed from both XTE J1550-564 and GRO J1655-40 at the transition (referred to by Kubota et al. as the "anomolous regime") between the classic thermal state in which the sources follow $L \propto T_{\text {in }}^{4}$ rather well, implying a constant $f_{\text {col }}$, and the higher luminosities at which they appear to deviate from this relation.

This is potentially of key importance, as it could provide an anchor from which to estimate the relative luminosity of Circinus ULX5, under the assumption that this transition occurs over a fairly narrow range of $L / L_{\mathrm{E}}$. Although this is a fairly strong assumption, as the behavior of Galactic BHBs can be quite diverse, XTE J1550-564 and GRO J1655-40 seem to make this transition at $L / L_{\mathrm{E}} \sim 0.3$ and 0.1 respectively (Gierliński \& Done 2004). Conservatively adopting the former for the 2001 $X M M-N e w t o n$ observation, we return to the previous estimate of $M_{\mathrm{BH}} \sim 90 M_{\odot}$. However, associating Circinus ULX5 with this accretion regime, rather than the canonical low/hard state, has the advantage that the $X M M-N e w t o n$ disk temperature is indeed lower than the temperatures observed from both XTE J1550-564 and GRO J1655-40 during the relevant transition $\left(T_{\text {in }} \sim 1 \mathrm{keV}\right)$, by roughly the factor expected for a $\sim 90 M_{\odot}$ black hole compared to a $\sim 10 M_{\odot}$ black hole. However, in the absence of any dynamical information on the putative binary system, and given the diversity in the behavior observed from Galactic BHB accretion disks (Gierliński \& Done 2004; Dunn et al. 2011; Reynolds \& Miller 2013), this mass estimate must still be considered speculative, and treated with the appropriate caution. Furthermore, we also urge caution in extrapolating the proposed identification of Circinus ULX5 to the general ULX population, as it is based on the specific behavior displayed by this source.

Such a black hole is just about at the upper limit of the mass range it is currently believed possible to form in situ via standard stellar evolution (Zampieri \& Roberts 2009; Belczynski et al. 2010). This would appear to require a low metallicity, $Z \sim$ $0.05 Z_{\odot}$ or less. The work by Oliva et al. (1999) does suggest the Circinus galaxy has a sub-solar metallicity, although at roughly $\sim 0.5 Z_{\odot}$ this may not be low enough to form such a black hole directly. If this mass is correct, more exotic formation mechanisms (e.g., Portegies Zwart et al. 2004) may be required. However, the metallicity estimates in Oliva et al. (1999) are based in circumnuclear clouds rather than the immediate environment around Circinus ULX5. Therefore, given that the mass is still ultimately uncertain, we defer detailed discussion of possible formation scenarios until more secure mass estimates and further studies focusing on the immediate environment of Circinus ULX5 are available. 
If our identification of the accretion regime displayed by Circinus ULX5 is correct, then in the most recent high flux state we are observing the re-emergence of the accretion disk as it again begins to dominate over the very high state corona, and in principle at lower luminosities the X-ray spectrum should also appear disk-dominated, as the source returns to the more traditional thermal state. Tao \& Blaes (2013) suggest the very high state corona could be related to the vertical structure of the disk at high luminosities, and are able to reproduce similar spectra to those observed with vertical dissipation profiles that dissipate more energy at larger scale heights, in the hot, ionized upper layers of the disk atmosphere. This disk state could also be described as having an energy dependent $f_{\text {col }}$, or alternatively, a distribution of $f_{\text {col }}$ values that produce the hard tail. This is potentially physically distinct from the corona that dominates during the low/hard state, which may well be associated with the base of a jet (Markoff et al. 2005; Miller et al. 2012; Reis \& Miller 2013).

In order to explain the observations of Circinus ULX5 as an extension of the high luminosity behavior of Galactic binaries, all of which are fully driven by an evolving disk structure and without invoking very large mid-plane disk temperatures, the evolution could roughly be along the following lines. As the source increases in luminosity from the classic thermal state, the characteristic height of the dissipation profile first increases relative to the scale height of the disc, such that more energy is dissipated in the scattering atmosphere and a strong, high energy tail emerges. Increasing the luminosity further still, the scale height of the disc also increases and in effect catches up with the dissipation profile, such that more of the energy is again dissipated in the optically thick regions of the disk, and the blackbody-like emission progressively dominates again.

Obtaining lower flux observations may therefore provide a simple test of our proposed identification. This would most likely require dedicated monitoring in order to identify and follow up periods of low flux. Although the temperature of the disk should decrease further, the evolution should switch to roughly following $L \propto T_{\text {in }}^{4}$, which would alleviate the rate of decrease, keeping the disk in the band observable with current soft X-ray instrumentation for a wide range of luminosities.

\subsection{Super-Eddington Accretion and X-Ray Outflows}

Galactic binaries in disk-dominated states frequently display evidence for outflows in the form of narrow, highly ionized iron absorption (e.g., Miller et al. 2006; Neilsen \& Lee 2009; Ponti et al. 2012; King et al. 2012). Furthermore, in agreement with basic expectations, the strength of these outflows appears to increase with increasing luminosity (Ponti et al. 2012). This prompted us to search for evidence of similar features in the joint XMM-Newton+NuSTAR data set obtained in 2013, which offers both the best photon statistics in the iron $K \alpha$ band, and the highest source luminosity of the higher quality data sets available (see Section 3.1.3).

Similar to our analysis of other bright ULXs (Walton et al. 2012a, 2013a), we do not find any statistically compelling narrow iron features in either absorption or emission, so in Figure 6 we present the equivalent width limits on any narrow lines that could have been present and remain undetected. Across the immediate Fe $\mathrm{K}$ bandpass (6-7 keV), any emission/ absorption lines must have $\mathrm{EW} \lesssim 50 \mathrm{eV}$. Although these limits are not as stringent as recently obtained for Holmberg IX X-1 (Walton et al. 2013a), in absolute terms they still require any lines to be weaker than the strongest features seen in Galactic
BHBs (King et al. 2012). Metallicity is likely to be an issue here, with Oliva et al. (1999) estimating the (circumnuclear) iron abundance to be $A_{\mathrm{Fe}} /$ solar $\sim 0.4$. However, even accounting for this iron abundance, the limits obtained still rule out the line strengths that might be expected from simple scaling of the features in e.g., GRS 1915+105 ( 30 eV; Neilsen \& Lee 2009) up to the Eddington ratio that would be inferred for a black hole of mass $\sim 10 M_{\odot}(\mathrm{EW} \gtrsim 200 \mathrm{eV})$. Thus, it seems likely that we cannot be viewing the central regions of Circinus ULX5 through any extreme super-Eddington outflow. Similar conclusions were drawn for Holmberg IX X-1 and NGC 1313 X-1. However, as noted earlier, we again stress that the local metallicity is not well constrained, which, if substantially lower than the circumnuclear metallicity, would further hinder line detection. Nevertheless, a larger black hole accreting at a lower Eddington rate also offers a plausible explanation for the lack of ionized absorption, as the solid angle subtended by outflows launched from the accretion disk is widely expected to increase with increasing Eddington luminosity (e.g., King 2009; Dotan \& Shaviv 2011; Kawashima et al. 2012). Thus, for a given observed luminosity, there should be a larger range of viewing angles that do not intercept any outflow launched for larger black hole masses.

In addition, any iron emission must be weaker than observed from many Galactic high mass X-ray binaries (HMXBs). Iron emission is ubiquitously observed from such sources (Torrejón et al. 2010), as they illuminate the strong stellar winds launched by their massive binary companions. Following the discussion outlined in Walton et al. (2013a) for Holmberg IX X-1, we argue that the lack of strong iron emission suggests that any stellar wind launched by the companion of Circinus ULX5 is probably not sufficient to power the observed X-ray luminosities via wind-fed accretion, and thus Circinus ULX5 most likely accretes via Roche-lobe overflow.

\section{CONCLUSIONS}

Prompted by a serendipitous detection with the NuSTAR observatory, we have undertaken a multi-epoch spectral and temporal analysis of an extremely luminous ULX located in the outskirts of the Circinus galaxy, utilizing data from most of the major X-ray observatories operating over the last decade, including coordinated follow-up observations with XMM-Newton and NuSTAR. Based on previous detections of ULX candidates in Circinus, we refer to this source Circinus ULX5. The NUSTAR data presented here represent one of the first instances of a ULX reliably detected at hard $(E>10 \mathrm{keV})$ X-rays. Circinus ULX5 is observed to vary on long time scales by at least a factor of $\sim 5$, and was caught in a historically bright state by our 2013 observations, with an observed $0.3-30.0 \mathrm{keV}$ luminosity of $1.6 \times 10^{40} \mathrm{erg} \mathrm{s}^{-1}$. During this epoch, the source displayed a curved 3-10 keV spectrum, broadly similar to other bright ULXs. We consider a variety of models for the broadband 0.3-30.0 keV spectrum obtained. Pure thermal models (direct accretion disk emission, cool optically thick Comptonization) result in a high energy excess in the NuSTAR data, and require a second emission component. However, this excess is too weak for the Compton reflection interpretation previously proposed for the 3-10 keV curvature in other ULXs.

In addition to the flux variability observed, Circinus ULX5 also displays strong spectral variability from epoch to epoch, and, at times, even within a single epoch. All the high quality data sets currently available are well modelled with a simple combination of a thermal accretion disk emission and a Comptonised corona, an interpretation which is further supported by 
the observed short-term variability properties. As the source luminosity increases, the accretion disk becomes more prominent. However, although the disk temperature and luminosity follow a common relation across all probed time scales, the observed relation is much shallower than the $L \propto T^{4}$ relation naively expected for blackbody radiation, varying instead as $L \propto T^{1.7}$. The spectral variability displayed by Circinus ULX5 is extremely reminiscent of that observed from the Galactic BHBs XTE J1550-564 and GRO J1655-40 at high luminosities, which also seem to roughly follow $L \propto T^{2}$. Identifying the lowest luminosity observation of Circinus ULX5 with the transition into the $L \propto T^{2}$ regime, as the spectral comparison would suggest, implies a black hole mass of $\sim 90 M_{\odot}$. This is also consistent with the lower disk temperature displayed by Circinus ULX5 during this epoch. However, we stress that given the fairly diverse behavior observed from Galactic BHB accretion disks, this mass estimate should be considered highly uncertain. Further study of this remarkable source is certainly warranted in order to see if this mass estimate truly holds up to scrutiny.

Finally, during the highest flux observation, we find no evidence for any iron features in either emission or absorption, similar again to other bright ULXs. Any features intrinsically present in the immediate Fe K bandpass must have $\mathrm{EW} \lesssim 50 \mathrm{eV}$. The implication is that we are not viewing the central regions of Circinus ULX5 through any extreme super-Eddington outflow, which would also be consistent with Circinus ULX5 hosting a relatively massive black hole.

The authors thank Koji Mukai for useful discussion regarding Galactic CVs, and Rubens Reis for discussion regarding Galactic BHBs. This research has made use of data obtained with the NUSTAR mission, a project led by the California Institute of Technology (Caltech), managed by the Jet Propulsion Laboratory (JPL) and funded by NASA, XMMNewton, an ESA science mission with instruments and contributions directly funded by ESA Member States and NASA, and the Suzaku observatory, a collaborative mission between the space agencies of Japan (JAXA) and the USA (NASA). In addition, this research has also made use of data obtained from NASA's Swift, Chandra, and Spitzer satellites. We thank the NUSTAR Operations, Software, and Calibration teams for support with the execution and analysis of these observations. This research has made use of the NUSTAR Data Analysis Software (NUSTARDAS) jointly developed by the ASI Science Data Center (ASDC, Italy) and Caltech (USA). We also made use of the NASA/IPAC Extragalactic Database (NED), which is operated by JPL, Caltech, under contract with NASA. Some of the figures included in this work have been produced with the Veusz plotting package: http://home.gna.org/veusz, written and maintained by Jeremy Sanders. F.E.B. acknowledges support from Basal-CATA (PFB-06/2007) and CONICYT-Chile (under grants FONDECYT 1101024 and Anillo ACT1101). M.B. wishes to acknowledge the support from the Centre National DÉtudes Spatiales (CNES).

\section{REFERENCES}

Abramowicz, M. A., Czerny, B., Lasota, J. P., \& Szuszkiewicz, E. 1988, ApJ, 332,646

Arnaud, K. A. 1996, in ASP Conf. Ser. 101, Astronomical Data Analysis Software and Systems V, ed. G. H. Jacoby \& J. Barnes (San Francisco, CA: ASP), 17

Bachetti, M., Rana, V., Walton, D. J., et al. 2013, ApJ, 778, 163

Barret, D. 2001, AdSpR, 28, 307

Bauer, F. E., Brandt, W. N., Sambruna, R. M., et al. 2001, AJ, 122, 182
Bauer, F. E., Dwarkadas, V. V., Brandt, W. N., et al. 2008, ApJ, 688, 1210

Belczynski, K., Bulik, T., Fryer, C. L., et al. 2010, ApJ, 714, 1217

Caballero-García, M. D., \& Fabian, A. C. 2010, MNRAS, 402, 2559

Cash, W. 1979, ApJ, 228, 939

Churazov, E., Gilfanov, M., \& Revnivtsev, M. 2001, MNRAS, 321, 759

Corongiu, A., Chiappetti, L., Haardt, F., et al. 2003, A\&A, 408, 347

Dauser, T., Wilms, J., Reynolds, C. S., \& Brenneman, L. W. 2010, MNRAS, 409, 1534

Davis, S. W., Blaes, O. M., Hubeny, I., \& Turner, N. J. 2005, ApJ, 621, 372

Done, C., Gierliński, M., \& Kubota, A. 2007, A\&ARv, 15, 1

Dotan, C., \& Shaviv, N. J. 2011, MNRAS, 413, 1623

Dunn, R. J. H., Fender, R. P., Körding, E. G., Belloni, T., \& Merloni, A. 2011, MNRAS, 411, 337

Edelson, R., Turner, T. J., Pounds, K., et al. 2002, ApJ, 568, 610

Fabian, A. C., Ross, R. R., \& Miller, J. M. 2004, MNRAS, 355, 359

Farrell, S. A., Webb, N. A., Barret, D., Godet, O., \& Rodrigues, J. M. 2009, Natur, 460, 73

Fazio, G. G., Hora, J. L., Allen, L. E., et al. 2004, ApJS, 154, 10

Fender, R., \& Belloni, T. 2012, Sci, 337, 540

Feng, H., \& Kaaret, P. 2006, ApJL, 650, L75

Feng, H., \& Soria, R. 2011, NAR, 55, 166

Finke, J. D., \& Böttcher, M. 2007, ApJ, 667, 395

For, B.-Q., Koribalski, B. S., \& Jarrett, T. H. 2012, MNRAS, 425, 1934

Freeman, K. C., Karlsson, B., Lynga, G., et al. 1977, A\&A, 55, 445

Garmire, G. P., Bautz, M. W., Ford, P. G., Nousek, J. A., \& Ricker, G. R., Jr 2003, Proc. SPIE, 4851, 28

Gehrels, N., Chincarini, G., Giommi, P., et al. 2004, ApJ, 611, 1005

Gierliński, M., \& Done, C. 2004, MNRAS, 347, 885

Gladstone, J. C., Roberts, T. P., \& Done, C. 2009, MNRAS, 397, 1836

Godet, O., Webb, N., Barret, D., et al. 2012, ATel, 4327, 1

Harrison, F. A., Craig, W. W., Christensen, F. E., et al. 2013, ApJ, 770, 103

Heida, M., Jonker, P. G., Torres, M. A. P., et al. 2013, MNRAS, 433, 681

Heil, L. M., Vaughan, S., \& Roberts, T. P. 2009, MNRAS, 397, 1061

Homan, J., Wijnands, R., van der Klis, M., et al. 2001, ApJS, 132, 377

Ishida, M., Tsujimoto, M., Kohmura, T., et al. 2011, PASJ, 63, 657

Jansen, F., Lumb, D., Altieri, B., et al. 2001, A\&A, 365, L1

Jonker, P. G., Heida, M., Torres, M. A. P., et al. 2012, ApJ, 758, 28

Jonker, P. G., \& Nelemans, G. 2004, MNRAS, 354, 355

Kajava, J. J. E., \& Poutanen, J. 2009, MNRAS, 398, 1450

Kalberla, P. M. W., Burton, W. B., Hartmann, D., et al. 2005, A\&A, 440, 775

Kawashima, T., Ohsuga, K., Mineshige, S., et al. 2012, ApJ, 752, 18

King, A. L., Miller, J. M., Raymond, J., et al. 2012, ApJL, 746, L20

King, A. R. 2009, MNRAS, 393, L41

Kong, A. K. H., Yang, Y. J., Yen, T.-C., Feng, H., \& Kaaret, P. 2010, ApJ, 722,1816

Koribalski, B. S., Staveley-Smith, L., Kilborn, V. A., et al. 2004, AJ, 128, 16

Koyama, K., Tsunemi, H., Dotani, T., et al. 2007, PASJ, 59, 23

Kubota, A., Done, C., \& Makishima, K. 2002, MNRAS, 337, L11

Kubota, A., \& Makishima, K. 2004, ApJ, 601, 428

Kubota, A., Makishima, K., \& Ebisawa, K. 2001a, ApJL, 560, L147

Kubota, A., Mizuno, T., Makishima, K., et al. 2001b, ApJL, 547, L119

Kuulkers, E., Norton, A., Schwope, A., \& Warner, B. 2006, X-Rays from Cataclysmic Variables (Cambridge: Cambridge Univ. Press), 421

Lasota, J.-P., Alexander, T., Dubus, G., et al. 2011, ApJ, 735, 89

Li, L.-X., Zimmerman, E. R., Narayan, R., \& McClintock, J. E. 2005, ApJS, 157,335

Liu, Q. Z., \& Mirabel, I. F. 2005, A\&A, 429, 1125

Makishima, K., Takahashi, H., Yamada, S., et al. 2008, PASJ, 60, 585

Markoff, S., Nowak, M. A., \& Wilms, J. 2005, ApJ, 635, 1203

Merloni, A., Fabian, A. C., \& Ross, R. R. 2000, MNRAS, 313, 193

Middleton, M. J., Cackett, E. M., Shaw, C., et al. 2012, MNRAS, 419, 336

Middleton, M. J., Miller-Jones, J. C. A., Markoff, S., et al. 2013, Natur, 493, 187

Middleton, M. J., Sutton, A. D., \& Roberts, T. P. 2011, MNRAS, 417, 464

Miller, J. M., Fabbiano, G., Miller, M. C., \& Fabian, A. C. 2003, ApJL, 585, L37

Miller, J. M., Pooley, G. G., Fabian, A. C., et al. 2012, ApJ, 757, 11

Miller, J. M., Raymond, J., Fabian, A., et al. 2006, Natur, 441, 953

Miller, J. M., Raymond, J., Fabian, A. C., et al. 2004, ApJ, 601, 450

Miller, J. M., Walton, D. J., King, A. L., et al. 2013, ApJL, 776, L36

Mineshige, S., Hirano, A., Kitamoto, S., Yamada, T. T., \& Fukue, J. 1994, ApJ, 426, 308

Mitsuda, K., Bautz, M., Inoue, H., et al. 2007, PASJ, 59, 1

Mitsuda, K., Inoue, H., Koyama, K., et al. 1984, PASJ, 36, 741

Mukai, K. 2005, in ASP Conf. Ser. 330, The Astrophysics of Cataclysmic Variables and Related Objects, ed. J.-M. Hameury \& J.-P. Lasota (San Francisco, CA: ASP), 147 
Nardini, E., Fabian, A. C., Reis, R. C., \& Walton, D. J. 2011, MNRAS, 410, 1251

Neilsen, J., \& Lee, J. C. 2009, Natur, 458, 481

Oliva, E., Marconi, A., \& Moorwood, A. F. M. 1999, A\&A, 342, 87

Piconcelli, E., Cappi, M., Bassani, L., Di Cocco, G., \& Dadina, M. 2003, A\&A, 412, 689

Ponti, G., Fender, R. P., Begelman, M. C., et al. 2012, MNRAS, 422, L11

Portegies Zwart, S. F., Baumgardt, H., Hut, P., Makino, J., \& McMillan, S. L. W. 2004, Natur, 428, 724

Poutanen, J., Lipunova, G., Fabrika, S., Butkevich, A. G., \& Abolmasov, P. 2007, MNRAS, 377, 1187

Ptak, A., Colbert, E., van der Marel, R. P., et al. 2006, ApJS, 166, 154

Raymond, J. C., \& Smith, B. W. 1977, ApJS, 35, 419

Reis, R. C., Fabian, A. C., \& Miller, J. M. 2010, MNRAS, 402, 836

Reis, R. C., \& Miller, J. M. 2013, ApJL, 769, L7

Reis, R. C., Miller, J. M., \& Fabian, A. C. 2009, MNRAS, 395, L52

Reis, R. C., Miller, J. M., Fabian, A. C., et al. 2011, MNRAS, 410, 2497

Remillard, R. A., \& McClintock, J. E. 2006, ARA\&A, 44, 49

Reynolds, M. T., \& Miller, J. M. 2013, ApJ, 769, 16

Risaliti, G., Harrison, F. A., Madsen, K. K., et al. 2013, Natur, 494, 449

Rivers, E., Markowitz, A., \& Rothschild, R. 2013, ApJ, 772, 114

Roberts, T. P. 2007, Ap\&SS, 311, 203

Ross, R. R., \& Fabian, A. C. 2005, MNRAS, 358, 211

Saito, K., Yamaoka, K., Fukuyama, M., et al. 2006, in Proc. VI Microquasar Workshop: Microquasars and Beyond, ed. T. Belloni (Trieste: SISSA), 93

Sale, S. E., Drew, J. E., Knigge, C., et al. 2010, MNRAS, 402, 713

Salvesen, G., Miller, J. M., Reis, R. C., \& Begelman, M. C. 2013, MNRAS, 431, 3510

Servillat, M., Farrell, S. A., Lin, D., et al. 2011, ApJ, 743, 6

Shakura, N. I., \& Sunyaev, R. A. 1973, A\&A, 24, 337

Shimura, T., \& Takahara, F. 1995, ApJ, 445, 780

Steiner, J. F., Narayan, R., McClintock, J. E., \& Ebisawa, K. 2009, PASP, 121,1279

Stern, D., Eisenhardt, P., Gorjian, V., et al. 2005, ApJ, 631, 163

Stobbart, A.-M., Roberts, T. P., \& Wilms, J. 2006, MNRAS, 368, 397
Strohmayer, T. E. 2009, ApJL, 706, L210

Strüder, L., Briel, U., Dennerl, K., et al. 2001, A\&A, 365, L18

Sutton, A. D., Roberts, T. P., Gladstone, J. C., et al. 2013, MNRAS, 434, 1702

Sutton, A. D., Roberts, T. P., Walton, D. J., Gladstone, J. C., \& Scott, A. E. 2012, MNRAS, 423, 1154

Swartz, D. A., Ghosh, K. K., Tennant, A. F., \& Wu, K. 2004, ApJS, 154, 519

Swartz, D. A., Soria, R., Tennant, A. F., \& Yukita, M. 2011, ApJ, 741, 49

Tao, T., \& Blaes, O. 2013, ApJ, 770, 55

Titarchuk, L. 1994, ApJ, 434, 570

Tomsick, J. A., Yamaoka, K., Corbel, S., et al. 2009, ApJL, 707, L87

Torrejón, J. M., Schulz, N. S., Nowak, M. A., \& Kallman, T. R. 2010, ApJ, 715,947

Tsujimoto, M., Guainazzi, M., Plucinsky, P. P., et al. 2011, A\&A, 525, A25

Turner, M. J. L., Abbey, A., Arnaud, M., et al. 2001, A\&A, 365, L27

Vaughan, S., Fabian, A. C., \& Nandra, K. 2003, MNRAS, 339, 1237

Walton, D. J., Gladstone, J. C., Roberts, T. P., et al. 2011a, MNRAS, 414, 1011

Walton, D. J., Miller, J. M., Harrison, F. A., et al. 2013a, ApJL, 773, L9

Walton, D. J., Miller, J. M., Reis, R. C., \& Fabian, A. C. 2012a, MNRAS, 426,473

Walton, D. J., Nardini, E., Fabian, A. C., Gallo, L. C., \& Reis, R. C. 2013b, MNRAS, 428, 2901

Walton, D. J., Reis, R. C., Cackett, E. M., Fabian, A. C., \& Miller, J. M. 2012b, MNRAS, 422, 2510

Walton, D. J., Reis, R. C., \& Fabian, A. C. 2010, MNRAS, 408, 601

Walton, D. J., Roberts, T. P., Mateos, S., \& Heard, V. 2011b, MNRAS, 416, 1844

Watarai, K., Mizuno, T., \& Mineshige, S. 2001, ApJL, 549, L77

Weisskopf, M. C., Brinkman, B., Canizares, C., et al. 2002, PASP, 114, 1

Werner, M. W., Roellig, T. L., Low, F. J., et al. 2004, ApJS, 154, 1

Williams, M. J., Bureau, M., \& Cappellari, M. 2010, MNRAS, 409, 1330

Wilms, J., Allen, A., \& McCray, R. 2000, ApJ, 542, 914

Winter, L. M., Mushotzky, R. F., \& Reynolds, C. S. 2006, ApJ, 649, 730

Zampieri, L., \& Roberts, T. P. 2009, MNRAS, 400, 677

Zdziarski, A. A., Poutanen, J., Paciesas, W. S., \& Wen, L. 2002, ApJ, 578, 357 